\title{
TU/e EmonOWEN

\section{All-optical logic based on ultrafast gain and index dynamics in a semiconductor optical amplifier}

\section{Citation for published version (APA):}

Dorren, H. J. S., Yang, X., Mishra, A. K., Li, Z., Ju, H., Waardt, de, H., Khoe, G. D., Simoyama, T., Ishikawa, H., Kawashima, H., \& Hasama, T. (2004). All-optical logic based on ultrafast gain and index dynamics in a semiconductor optical amplifier. IEEE Journal of Selected Topics in Quantum Electronics, 10(5), 1079-1092. https://doi.org/10.1109/JSTQE.2004.835293

DOI:

10.1109/JSTQE.2004.835293

Document status and date:

Published: 01/01/2004

\section{Document Version:}

Publisher's PDF, also known as Version of Record (includes final page, issue and volume numbers)

\section{Please check the document version of this publication:}

- A submitted manuscript is the version of the article upon submission and before peer-review. There can be important differences between the submitted version and the official published version of record. People interested in the research are advised to contact the author for the final version of the publication, or visit the $\mathrm{DOI}$ to the publisher's website.

- The final author version and the galley proof are versions of the publication after peer review.

- The final published version features the final layout of the paper including the volume, issue and page numbers.

Link to publication

\section{General rights}

Copyright and moral rights for the publications made accessible in the public portal are retained by the authors and/or other copyright owners and it is a condition of accessing publications that users recognise and abide by the legal requirements associated with these rights.

- Users may download and print one copy of any publication from the public portal for the purpose of private study or research.

- You may not further distribute the material or use it for any profit-making activity or commercial gain

- You may freely distribute the URL identifying the publication in the public portal.

If the publication is distributed under the terms of Article 25fa of the Dutch Copyright Act, indicated by the "Taverne" license above, please follow below link for the End User Agreement:

www.tue.nl/taverne

Take down policy

If you believe that this document breaches copyright please contact us at:

openaccess@tue.nl

providing details and we will investigate your claim. 


\title{
All-Optical Logic Based on Ultrafast Gain and Index Dynamics in a Semiconductor Optical Amplifier
}

\author{
H. J. S. Dorren, Xuelin Yang, Arvind K. Mishra, Zhonggui Li, Heongkyu Ju, Huug de Waardt, \\ Giok-Djan Khoe, Fellow, IEEE, Takasi Simoyama, Hiroshi Ishikawa, Fellow, IEEE, Hitoshi Kawashima, and \\ Toshifumi Hasama
}

Invited Paper

\begin{abstract}
We investigate nonlinear carrier dynamics in a multiquantum-well semiconductor optical amplifier (SOA) in the context of ultrafast all-optical logic. A rate-equation model is presented that accounts for two-photon absorption, free-carrier absorption, self- and cross phase modulation, carrier heating, spectral, spatial hole burning, and self- and cross polarization modulation. The nonlinear refractive index dynamics is investigated theoretically and experimentally. We find nonlinear phase changes larger than $\pi$ radians, which recovers on a timescale in the order of $1 \mathrm{ps}$. We also investigate a nonlinear AND gate that consists of an SOA that is placed in an asymmetric Mach-Zehnder interferometer. We show that the gate can be operated using 800-fJ optical pulses with duration of 200 fs while having a contrast ratio larger than $11 \mathrm{~dB}$.
\end{abstract}

Index Terms-Optical logic, optical signal processing ultrafast carrier dynamics, semiconductor optical amplifier.

\section{INTRODUCTION}

D IGITAL optical techniques are expected to become increasingly important in futuristic ultrahigh capacity telecommunication networks. Optical communications systems with a capacity of hundreds of gigabits per second are commercially available today, and the capacity has been pushed above $10 \mathrm{~Tb} / \mathrm{s}$ in research laboratories. The highest bit rate per channel that has been reached is greater than $1 \mathrm{~Tb} / \mathrm{s}$ [1].

For data rates greater than $40 \mathrm{~Gb} / \mathrm{s}$, transmission systems often use optical techniques, since direct electronic processing is limited in speed. In optical time-domain multiplexed systems, the data at a transmission channel are demultiplexed to a lower bit rate using a clocked optical switch. Such an optical switch

Manuscript received January 19, 2004; revised June 10, 2004. The work of the COBRA researchers was supported by the Netherlands Foundation of Scientific Research (NWO), the Technology Foundation STW, and the Ministry of Economic Affairs through the NRC Photonics grant and the Innovational Research Incentives Scheme program. The work of the AIST and FESTA researchers was supported in part by the Ministry of Economy, Trade and Industry of Japan and in part by the New Energy and Industrial Technology development association within the framework of the Femtosecond Technology project.

H. J. S. Dorren, X. Yang, A. K. Mishra, Z. Li, H. Ju, H. de Waardt and G. D. Khoe are with the COBRA Research Institute, Eindhoven University of Technology, Eindhoven 5600 MB, The Netherlands (E-mail: H.J.S.Dorren@tue.nl).

T. Simoyama and H. Ishikawa are with the the Femtosecond Technology Research Association (FESTA), Tsukuba 300-2635, Japan.

H. Kawashima and T. Hasama are with the Photonics Research Institute, National Institute of Advanced Industrial Science and Technology (AIST), Tsukuba 305-8568, Japan.

Digital Object Identifier 10.1109/JSTQE.2004.835293 can be realized by employing photo-generated changes in carrier density of semiconductor optical amplifiers (SOAs). SOAs are attractive as such a nonlinear element, since they provide a high gain and exhibit a strong refractive-index change and allow photonic integration [2].

To obtain optical switching, the SOA is usually placed in an interferometer. Optical switching in the picosecond regime has been shown using a variety of configurations such as the symmetric Mach-Zehnder (SMZ) interferometer, the ultrafast nonlinear interferometer (UNI), and the terahertz optical asymmetric demultiplexer (TOAD) [3]-[8]

In most applications, SOAs are operated by utilizing nonlinearities introduced by resonant optical transitions. The speed of all-optical switches based on SOA nonlinearities is determined by the carrier dynamics of the SOA. In particular, if resonant transitions are employed by using photons with an energy corresponding to the band edge, the switching speed is restricted by the carrier lifetime, which determines the speed that carriers can be injected in the active region. Faster operation can be obtained by artificially reducing the carrier lifetime by using an additional holding beam [9], [10]. Also, it has been shown by using photons with a photon energy below the bandgap that the SOA gain recovery time is determined by the length of the control pulse due to the instantaneous two-photon-absorption (TPA) transient [11], [12]. It should be noted however, the corresponding refractive index recovery is slower, since, due to the Kramers-Kronig relationship, the refractive index also depends on the gain at other wavelengths [12].

The optical switching configurations mentioned above have in common that they allow operation at high repetition rates by using differential interference effects to overcome the speed limitations imposed by the carrier lifetime, while still utilizing resonant nonlinearities. It has been shown by combining switches based on nonlinear interference effects that more sophisticated all-optical logic functionalist can be realized. In particular, proof of concept has been given for optical address recognizers [13]-[15], optical bit-level synchronizers [16], [17], optical clock recovery circuits [18], optical shift registers with inverters [19], [20], optical memories with read-write abilities [21]-[24], binary half- and full adders [25], [26], pseudorandom number generators [27], optical parity checkers [28], and optical packet switches [29]. 
The processing speed of the digital optical logic based on resonant optical transitions as described above is restricted to approximately $200 \mathrm{GHz}$ [2]. The reason for this is that the SOA recovery depends on the carrier lifetime which makes it so that the SOA fully recovers in approximately $1 \mathrm{~ns}$. Differential operation can partly overcome this issue but at switching rates higher than $200 \mathrm{GHz}$ [2], the SOA carrier population cannot establish an equilibrium Fermi-Dirac distribution within one bit-time [30].

Meanwhile, research has been carried out to investigate SOA gain and index dynamics at subpicosecond timescales. Early experiments indicated that strong nonlinearities on sub picosecond timescales in bulk and multiquantum-well (MQW) SOAs can take place [30]-[34]. These nonlinearities were soon identified to be related with nonequilibrium changes in the carrier energy distribution [31], [35]. The main nonlinear processes responsible for these nonequilibrium changes were identified to be spectral hole burning, carrier heating and TPA [36]-[38]. Early experimental results on nonlinear index changes on subpicosecond timescales were published in [12], [39]-[41]. Rateequation models that describe nonlinear carrier dynamics on subpicosecond timescales were firstly published in [36], [37], [42], [43]. Later microscopic models that describe the SOA carrier dynamics on subpicosecond timescales have been published [44], [45].

Switching experiments, employing femtosecond pulses in an ultrafast nonlinear interferometer were reported in [46]-[48]. Wavelength switching experiments employing ultrashort pulses were published in [49]-[51]. In this paper, we investigate nonlinear carrier dynamics in an MQW SOA in the context of ultrafast all-optical logic. The nonlinear carrier dynamics of an MQW SOA is investigated using a rate-equation model that accounts for TPA, free-carrier absorption (FCA), self- and crossphase modulation, carrier heating, spectral, spatial hole burning, and self- and cross-polarization modulation. In particular, attention is paid to the nonlinear refractive index dynamics that is also investigated experimentally. We find using an experimental approach related to spectral interferometry, nonlinear phase changes in an MQW SOA larger than $\pi$ radians that recover on a timescale in the order of $1 \mathrm{ps}$. We also investigate a nonlinear AND gate that consists of a single MQW SOA that is placed in an SMZ interferometer. We show that the gate can be operated using 800 -fJ optical pulses with duration of $200 \mathrm{fs}$ while having a contrast ratio larger than $11 \mathrm{~dB}$.

\section{THEORY}

A rate-equation model that describes polarization dependent nonlinear gain and index dynamics in SOAs on subpicosecond timescales is presented in [52]. This model takes into consideration carrier dynamics on femtosecond timescales driven by TPA and FCA, and accounts for self- and cross-phase modulation, carrier heating, and spectral and spatial hole burning as well as self- and cross-polarization modulation. As shown in [24], the polarization dependent gain saturation is taken into account by assuming that the polarized optical field can be decomposed into transverse electric (TE) and transverse magnetic (TM) components that propagate "independently" through the SOA, although they have indirect interaction with each other via the gain saturation. We have accounted for different TE and TM gains by assuming that these polarizations couple to different hole reservoirs. This assumption is justified by the fact that in zinc-blende structures such as GaAs and InP, the optical transitions occur between an $l=0$ type conduction band state and a (degenerate) $l=1$ type valence band state. Two out of the three possible transition types are selected by the TE and TM polarizations with the two corresponding inversions. In the isotropic bulk situation, these two transitions will occur in a fully symmetric manner, but we are now interested in the case where tensile strain is built into the bulk medium, and this will cause an asymmetry between the two transition types, such that TM will be favored over TE transitions. The model does not account for the propagation of hybrid modes and for polarization dependence introduced by the device structure.

The incoming arbitrarily polarized electric fields is decomposed in a component parallel to the layers in the waveguide ( $x$ component, TE mode) and a perpendicular component ( $y$ component, TM mode). These two polarization directions are along the principal axes $(\hat{x}, \hat{y})$ that diagonalize the wave propagation in the SOA. In fact, apart from their indirect interaction through the carrier dynamics in the device, these two polarizations propagate independently from each other. The total electric field is given by

$$
\begin{aligned}
E^{\mathrm{TE} / \mathrm{TM}}(z, t)= & {\left[A^{\mathrm{TE}}(z, t) \hat{x}+A^{\mathrm{TM}}(z, t) \hat{y}\right] e^{i\left(\omega_{0} t-k_{0} z\right)} } \\
& +c . c . ; \\
S^{\mathrm{TE} / \mathrm{TM}}(z, t)= & \left|A^{\mathrm{TE} / \mathrm{TM}}(z, t)\right|^{2}
\end{aligned}
$$

where $k_{0}=\left(n\left(\omega_{0}\right) / c\right) \omega_{0}, n\left(\omega_{0}\right)$ is the refractive index taken at the central frequency $\omega_{0}$ and $c$ the light velocity in vacuum, and $\hat{x}$ and $\hat{y}$ are unit vectors along the $x$ and $y$ directions. The frequency $\omega_{0}$ has been chosen such that the complex pulse amplitudes $A^{\mathrm{TE} / \mathrm{TM}}(z, t)$ are slowly varying functions of $z$ and $t$. The propagation equations for the TE and TM modes are

$$
\begin{aligned}
\left(\frac{\partial}{\partial z}+\frac{1}{v_{g}} \frac{\partial}{\partial t}\right) A^{\mathrm{TE}}(z, t)=\{ & \frac{1}{2} \Gamma^{\mathrm{TE}}(1+i \alpha) g^{\mathrm{TE}}(z, t) \\
& -\frac{1}{2} \alpha_{\mathrm{int}}-\frac{1}{2} \Gamma_{2} \beta_{2}\left(1+i \alpha_{2}\right) \\
& \times\left[S^{\mathrm{TE}}(z, t)+S^{\mathrm{TM}}(z, t)\right] \\
& -\frac{1}{2} \Gamma^{\mathrm{TE}} \beta_{c} n_{c}(z, t) \\
& \left.-\frac{1}{2} \Gamma^{\mathrm{TE}} \beta_{v} n_{x}(z, t)\right\} \\
& \times A^{\mathrm{TE}}(z, t) \\
\left(\frac{\partial}{\partial z}+\frac{1}{v_{g}} \frac{\partial}{\partial t}\right) A^{\mathrm{TM}}(z, t)=\{ & \frac{1}{2} \Gamma^{\mathrm{TM}}(1+i \alpha) g^{\mathrm{TM}}(z, t) \\
& -\frac{1}{2} \alpha_{\mathrm{int}}-\frac{1}{2} \Gamma_{2} \beta_{2}\left(1+i \alpha_{2}\right) \\
& \times\left[S^{\mathrm{TE}}(z, t)+S^{\mathrm{TM}}(z, t)\right] \\
& -\frac{1}{2} \Gamma^{\mathrm{TM}} \beta_{c} n_{c}(z, t) \\
& \left.-\frac{1}{2} \Gamma^{\mathrm{TM}} \beta_{v} n_{y}(z, t)\right\} \\
& \times A^{\mathrm{TM}}(z, t)
\end{aligned}
$$


TABLE I

SOA PARAMETER DEFINITIONS AND THEIR VALUES

\begin{tabular}{|c|c|c|c|}
\hline Parameter & Symbol & Value & Units \\
\hline Active volume & $V=L \times W \times D$ & $750 \times 2 \times 0.1$ & $\mu m^{3}$ \\
\hline Confinement factor & $\Gamma_{T E}, \Gamma_{T M}, \Gamma_{2}$ & $0.032,0.021,0.09$ & \\
\hline Phase modulation coefficients & $\alpha, \alpha_{2}$ & $1.2,-1.5$ & \\
\hline FCA coefficients & $\beta_{c}, \beta_{v}$ & $1 \times 10^{-10}, 0$ & $\mu m^{2}$ \\
\hline Electron-hole pair lifetime & $\tau_{s}$ & 1.3 & $n s$ \\
\hline Gain coefficient & $a_{T E}\left(\omega_{0}\right), a_{T M}\left(\omega_{0}\right)$ & $7.0 \times 10^{-5}, 5.5 \times 10^{-5}$ & $\mu m^{3} / p s$ \\
\hline Group velocity & $v_{g}$ & 100 & $\mu m / p s$ \\
\hline Internal loss & $\alpha_{\text {int }}$ & 0.00175 & $\mu m^{-1}$ \\
\hline Optical transition energies & $E_{c}, E_{2 c}$ & $0.03,0.7$ & $\mathrm{eV}$ \\
\hline \multicolumn{4}{|l|}{ (Valence band) } \\
\hline Optical transition energies & $E_{v}, E_{2 v}$ & $0.003,0.07$ & $\mathrm{eV}$ \\
\hline \multicolumn{4}{|l|}{ (Conduction band) } \\
\hline Carrier-carrier scattering times & $\tau_{l c}, \tau_{l v}$ & $0.1,0.05$ & ps \\
\hline Carrier-phonon relaxation times & $\tau_{h c}, \tau_{h v}$ & $0.7,0.25$ & $p s$ \\
\hline TPA coefficient & $\beta_{2}$ & $2.5 \times 10^{-9}$ & $\mu m^{2}$ \\
\hline Optical transition state density & $N_{0}$ & $1.25 \times 10^{6}$ & $\mu m^{-3}$ \\
\hline
\end{tabular}

where the SOA parameters and their physical definitions are listed in Table I. The first term on the right-hand side of (2) and (3) represents the linear gain. $\alpha$ is the phase modulation parameter (or linewidth enhancement factor). The second term represents the TPA that is modeled by assuming that both the TE and TM modes are involved in the TPA process, where $\alpha_{2}$ is the corresponding phase modulation parameter, while the last two terms represent the FCA in the conduction and valence bands. The complex field amplitudes can be related to the intensities $S^{\mathrm{TE} / \mathrm{TM}}(z, t)$ and the phases $\phi^{\mathrm{TE} / \mathrm{TM}}(z, t)$ through the well-known relationship $A^{\mathrm{TE} / \mathrm{TM}}(z, t)=\sqrt{S^{\mathrm{TE} / \mathrm{TM}}(z, t)} e^{i \phi^{\mathrm{TE} / \mathrm{TM}}(z, t)}$. The gains for both modes can be expressed as

$$
\begin{aligned}
& g^{\mathrm{TE}}(z, t)=\frac{1}{v_{g}} a^{\mathrm{TE}}\left(\omega_{0}\right)\left[n_{c}(z, t)+n_{x}(z, t)-N_{0}\right] \\
& g^{\mathrm{TM}}(z, t)=\frac{1}{v_{g}} a^{\mathrm{TM}}\left(\omega_{0}\right)\left[n_{c}(z, t)+n_{y}(z, t)-N_{0}\right]
\end{aligned}
$$

where $a^{\mathrm{TE} / \mathrm{TM}}\left(\omega_{0}\right)$ are the gain coefficients. Our model takes into account three bands. The numbers of electrons that are involved in the optical transition in the conduction band is $n_{c}(z, t)$. We have two reservoirs of holes identified with index $x$ and $y$. Holes identified with $x$ couple with electrons through TE polarized light. The number of holes that can participate in this transition is $n_{x}(z, t)$. Similarly, holes identified with $y$ couple with electrons through TM polarized light. The corresponding reservoir of holes that can participate in the optical transition is $n_{y}(z, t) . N_{0}$ is the total number of states involved in the stimulated emission. The carrier densities satisfy

$$
\begin{aligned}
\frac{\partial n_{c}(z, t)}{\partial t}= & -\frac{n_{c}(z, t)-\bar{n}_{c}(z, t)}{\tau_{1 c}} \\
& -v_{g} g^{\mathrm{TE}}(z, t) S^{\mathrm{TE}}(z, t) \\
& -v_{g} g^{\mathrm{TM}}(z, t) S^{\mathrm{TM}}(z, t) \\
& -n_{c}(z, t) \beta_{c} v_{g}\left[S^{\mathrm{TE}}(z, t)+S^{\mathrm{TM}}(z, t)\right] \\
\frac{\partial n_{x}(z, t)}{\partial t}= & -\frac{n_{x}(z, t)-\bar{n}_{x}(z, t)}{\tau_{1 v}} \\
& -v_{g} g^{\mathrm{TE}}(z, t) S^{\mathrm{TE}}(z, t) \\
& -n_{x}(z, t) \beta_{v} v_{g}\left[S^{\mathrm{TE}}(z, t)+S^{\mathrm{TM}}(z, t)\right] \\
\frac{\partial n_{y}(z, t)}{\partial t}= & -\frac{n_{y}(z, t)-\bar{n}_{y}(z, t)}{\tau_{1 v}} \\
& -v_{g} g^{\mathrm{TM}}(z, t) S^{\mathrm{TM}}(z, t) \\
& -n_{y}(z, t) \beta_{v} v_{g}\left[S^{\mathrm{TE}}(z, t)+S^{\mathrm{TM}}(z, t)\right] .
\end{aligned}
$$


The first terms on the right-hand sides of (6)-(8) describe the relaxation of the electrons and holes to their quasi-equilibrium values $\bar{n}_{i}(z, t), i \in\{c, x, y\}$ that are specified later. These relaxation processes are driven by the electron-electron and hole-hole interactions with typical timescales of 50-100 fs. The second terms describe the stimulated emission, and the third term describes FCA. Before the SOA model can be completed, we need to formulate the equation for the electron-hole pair density $N(z, t)$

$$
\begin{aligned}
\frac{\partial N(z, t)}{\partial t}= & \frac{I}{e V}-\frac{N}{\tau_{s}} \\
& -v_{g}\left[g^{\mathrm{TE}}(z, t) S^{\mathrm{TE}}(z, t)+g^{\mathrm{TM}}(z, t) S^{\mathrm{TM}}(z, t)\right] \\
& +v_{g} \beta_{2}\left[S^{\mathrm{TE}}(z, t)+S^{\mathrm{TM}}(z, t)\right]^{2}
\end{aligned}
$$

where it is noted that $N(z, t)$ counts all the electron-hole pairs, including those that are not directly available for stimulated emission. The energy densities satisfy

$$
\begin{aligned}
\frac{\partial U_{c}(z, t)}{\partial t}= & \beta_{c} \hbar \omega_{0} n_{c}(z, t) v_{g}\left[S^{\mathrm{TE}}(z, t)+S^{\mathrm{TM}}(z, t)\right] \\
& -E_{c} v_{g}\left[g^{\mathrm{TE}}(z, t) S^{\mathrm{TE}}(z, t)\right. \\
& \left.+g^{\mathrm{TM}}(z, t) S^{\mathrm{TM}}(z, t)\right] \\
& +E_{2 c} v_{g} \beta_{2}\left[S^{\mathrm{TE}}(z, t)+S^{\mathrm{TM}}(z, t)\right]^{2} \\
& -\frac{U_{c}(z, t)-\bar{U}_{c}(z, t)}{\tau_{h c}} \\
\frac{\partial U_{v}(z, t)}{\partial t}= & \beta_{v} \hbar \omega_{0} v_{g}\left[n_{x}(z, t)+n_{y}(z, t)\right] \\
& \times\left[S^{\mathrm{TE}}(z, t)+S^{\mathrm{TM}}(z, t)\right] \\
& -E_{v} v_{g}\left[g^{\mathrm{TE}}(z, t) S^{\mathrm{TE}}(z, t)\right. \\
& \left.+g^{\mathrm{TM}}(z, t) S^{\mathrm{TM}}(z, t)\right] \\
& +E_{2 v} v_{g} \beta_{2}\left[S^{\mathrm{TE}}(z, t)+S^{\mathrm{TM}}(z, t)\right]^{2} \\
& -\frac{U_{v}(z, t)-\bar{U}_{v}(z, t)}{\tau_{h v}}
\end{aligned}
$$

where $U_{c}(z, t)$ represents the energy density in the conduction band and $U_{v}(z, t)$ the overall energy density in the valence band. In these equations, the first terms describe the change in energy density due to FCA. The second terms describe the contribution of the stimulated emission, and the third terms account for the TPA. The last term represent the relaxation to equilibrium due to carrier-phonon interactions.

The carrier density $N(z, t)$ and the energy density $U_{c}(z, t)$ are needed to self-consistently calculate in each time step the quasi-Fermi level $E_{f c}(z, t)$ and temperature $T_{c}(z, t)$ of the electrons, using

$$
\begin{aligned}
N(z, t) & =\frac{1}{V} \sum_{k} F\left(E_{f_{c}}(z, t), T_{c}(z, t), \frac{\hbar^{2} k^{2}}{2 m_{c}^{*}}\right) \\
U_{c}(z, t) & =\frac{1}{V} \sum_{k} \frac{\hbar^{2} k^{2}}{2 m_{c}^{*}} F\left(E_{f c}(z, t), T_{c}(z, t), \frac{\hbar^{2} k^{2}}{2 m_{c}^{*}}\right)
\end{aligned}
$$

where $F(\mu, T, E)$ is the Fermi-Dirac distribution function defined as

$$
F(\mu, T, E)=\frac{1}{\left[1+\exp \left(\frac{E-\mu}{k T}\right)\right]} .
$$

A similar procedure can be used to compute the Fermi levels and temperatures in the valence band, but one has to correct with a factor of two, since there are two subbands involved. The quasi-equilibrium values $\bar{n}_{i}(z, \tau)$ are given by

$$
\begin{aligned}
\bar{n}_{c}(z, t) & =N_{0} F\left(E_{f c}(z, t), T_{c}(z, t), \frac{\hbar^{2} k^{2}}{2 m_{c}^{*}}\right) \\
\bar{n}_{x}(z, t) & =f \bar{n}_{y}(z, t) \\
& =\frac{f N_{0}}{1+f} F\left(E_{f v}(z, t), T_{v}(z, t), \frac{\hbar^{2} k^{2}}{2 m_{v}^{*}}\right)
\end{aligned}
$$

and the quasi-equilibrium values $\bar{U}_{c, v}(z, t)$ are

$$
\bar{U}_{v, c}(z, t)=\frac{1}{V} \sum_{k} \frac{\hbar^{2} k^{2}}{2 m_{v, c}^{*}} F\left(E_{f v, c}(z, t), T_{L}, \frac{\hbar^{2} k^{2}}{2 m_{v, c}^{*}}\right)
$$

where $T_{L}$ is the lattice temperature and $f$ is the population imbalance factor describing the gain anisotropy introduced by tensile strain in the SOA. Equation (16) describes how the equilibrium populations $\bar{n}_{x}(z, \tau)$ and $\bar{n}_{y}(z, \tau)$ are clamped to each other as a consequence of tensile strain [24]. In case of unstrained bulk material, the gain will be isotropic and $f=1$. In case of tensile strain, TM gain will be larger than TE, i.e., $f<1$. For the energy relaxation, the temperature must be taken equal to the lattice temperature $T_{L}(300 \mathrm{~K})$.

We are aware of the fact that neglecting the gain and group velocity dispersion in our model for pulse propagation in an SOA limits the applicability of our model [53], [54]. When the pulse duration is as short as 50-100 fs, we can no longer expect good agreement between our model and measurements. However, in view of the apparently successful application of earlier models for pulses as short as $200 \mathrm{fs}$, we expect that the present model should be applicable for pulses of the same duration, especially when the central pulse frequency coincides with the gain maximum [36], [37], [42], [55]. Using the gain curves of the SOA that are used in our experiments [56], we have estimated, assuming a pulse with a spectral width of $20 \mathrm{~nm}$, that for a 750- $\mu \mathrm{m}$-long amplifier, only minor changes occur in the pulse shape. It should be remarked, however, that large distortions on the pulse shape could be expected if the central pulse frequency is close to or at the zero gain region (at transparency). In this case, large changes in the pulse shape take place, which could even lead to pulse breakup [57].

Moreover, we want to remark that we have modeled the phase change by using a constant linewidth enhancement factor that is defined in the usual way [53], [55]. Although this ignores dispersive effects due to strong variations in the carrier density, such a treatment leads to result that are in agreement with experimental results.

Finally, we did not account for different group velocity dispersion coefficients for the TE and TM modes. Numerical simulations show that the pulse broadening due to group velocity dispersion is approximately $5 \%$ for a $500-\mu \mathrm{m}$-long SOA, which 


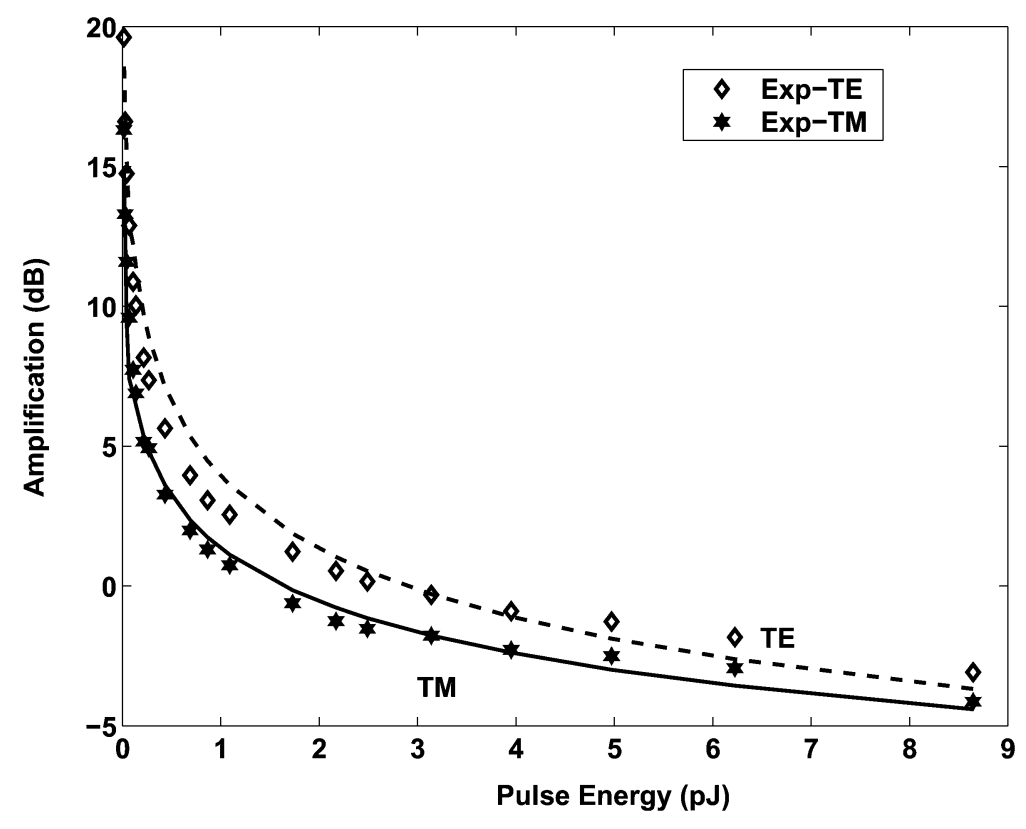

Fig. 1. Measured and computed polarization dependent amplification for the TE and TM modes as a function of the pump pulse energy. The diamond-shaped points represents the measured amplification for the TE mode and the star-shaped points represent the measured data for the TM mode. The dashed line represents the computed result for the TE mode and the solid line represents the computed result for the TM mode. The SOA injection current was $200 \mathrm{~mA}$ and in the computations, we used $f=0.8$.

is negligible with respect to the pulse broadening due to gain saturation It is shown in [58] that the difference in the group velocity between the TE and TM modes is in the order of $1 \%$. We estimate that for a $500-\mu \mathrm{m}$-long SOA, the pulse distortion effects remain far below $10 \%$. This implies that for a not too long amplifier pulse pattern effects due to birefringence of the SOA are negligible.

\section{SimULATION RESULtS}

The set of (2)-(17) is solved numerically. In our simulations, the SOA length is $750 \mu \mathrm{m}$, and the active volume of $150 \mu \mathrm{m}^{3}$. We will consider optical pulses with Gaussian shape [200 fs, full-width at half-maximum (FWHM)] as input. The SOA pump current is $120 \mathrm{~mA}$. All other SOA parameters are listed in Table I. These parameters characterize the SOA used in the experiments in the sections that follow. The confinement factor $\Gamma^{\mathrm{TM}}$ is chosen to be $30 \%$ less than $\Gamma^{\mathrm{TE}}$ (see Table I) [58], [59].

It should be noted, however, that the SOA parameters $N_{0}$, $G^{\mathrm{TE} / \mathrm{TM}}, \alpha_{\mathrm{int}}^{\mathrm{TE} / \mathrm{TM}}$, and $v_{g}^{\mathrm{TE} / \mathrm{TM}}$ cannot be estimated accurately. We have solved this problem by compensating the combined uncertainties in these parameter values by assigning values to $f$ and $a^{\mathrm{TE} / \mathrm{TM}}\left(\omega_{0}\right)$ in such a way that the SOA gain corresponds to typical values. In the simplest approach, one would choose $a^{\mathrm{TE}}\left(\omega_{0}\right)=a^{\mathrm{TM}}\left(\omega_{0}\right)$, which is correct in case of isotropic gain, whereas $f$ can be estimated from the measured TE and TM amplification curves by using (4) and (5) and (15) and (16). In this case, the polarization dependent gain could be totally explained by the band filling effects that are represented by the factor $f$. In a somewhat more complicated approach, one can assume different values for $a^{\mathrm{TE}}\left(\omega_{0}\right)$ and $a^{\mathrm{TM}}\left(\omega_{0}\right)$ as in [24] for a continuous-wave analysis. The difficulties in estimating $f$ and $a^{\mathrm{TE} / \mathrm{TM}}\left(\omega_{0}\right)$ may be inherent to our modeling the SOA strain in terms of the population imbalance factor $f$. In a more accurate, but also much more complicated, model, one can calculate the band structure and transition matrix elements in the presence of tensile strain and keep track of the different optical transitions involved as well as the relevant populations. This would, however, extend beyond the scope of the present approach [60], [61].

The TPA coefficient has been chosen in such a way that the SOA gain saturation is in agreement with experimental results presented in [57]. In Fig. 1, the SOA gain is presented as a function of the pulse energy. It follows from Fig. 1 that the net amplification becomes negative for pulse energies larger than $3 \mathrm{pJ}$ in the TE mode and $1.8 \mathrm{pJ}$ in the TM mode. The curve presented in Fig. 1 is in quantitative agreement with experimental results presented in [57]. The net attenuation is due to the combined effects of TPA and FCA. It follows from Fig. 1 that for large pulse energies, the difference in TE and TM gain almost vanishes, which can be explained by the fact that both modes equally contribute to the TPA. Thus, for high energetic optical pulses, the TPA terms in (2) and (3) dominate.

In the following numerical experiments, we investigate the feasibility of polarization switching using several pump-probe configurations. We will consider two 200 -fs optical pulses that copropagate through the SOA for two different situations. In the first case, the pump pulse is either TE or TM polarized. In the second case the pump pulse is linearly polarized under an angle of $45^{\circ}$ with the TE and TM polarization axes. While the pulse travels through the SOA, not only the TE and TM field component intensities will be amplified (or attenuated), but also their phase difference will change. Hence, the state of polarization changes dynamically during the propagation of the pulse through the SOA. These processes are fully described in (2) and (3). The pump pulses have variable pulse energy. The probe pulse is linearly polarized (TE or TM) or under an angle of $45^{\circ}$ with respect to the TE and TM polarization axes. The total probe 


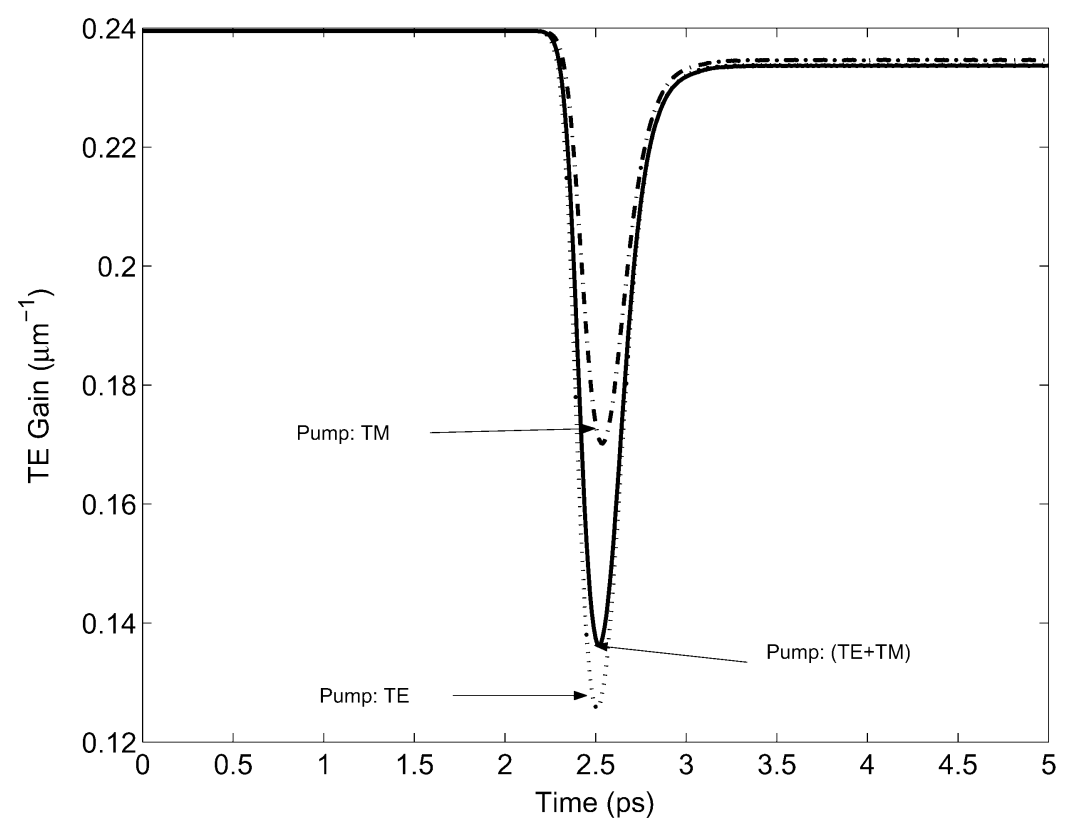

(a)

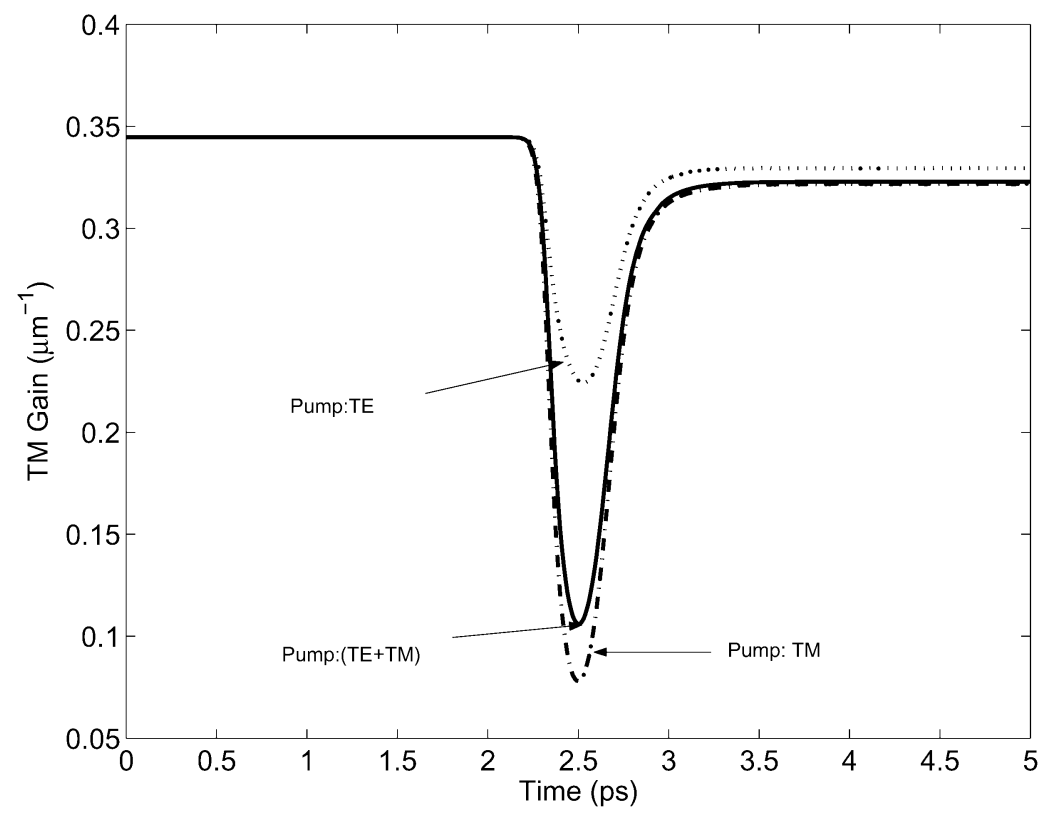

(b)

Fig. 2. Time variation of gain at the middle of SOA (at $250 \mu \mathrm{m}$ ), where the pulse energy is $1.7 \mathrm{pJ}$, and the pulse polarizations are the same as Fig. 1. (a) TE gain. (b) TM gain.

pulse energy is fixed to $0.8 \mathrm{fJ}$. This small energy guarantees that the probe pulse propagate linearly through the SOA. The delay between the pump and probe pulses is optimized to be around 5 fs such that the latter propagates in the gain minimum introduced by the pump pulse.

Fig. 2 shows the gain variations in the middle of the $\operatorname{SOA}(z=$ $250 \mu \mathrm{m})$ for the different polarization modes. It can be observed that initially the SOA gains $g^{\mathrm{TE} / \mathrm{TM}}$ are 0.24 and $0.34 \mu \mathrm{m}^{-1}$ for the TE and TM modes respectively. As an example, in Fig. 2(a), the TE gain is presented for different pump polarizations, where the pump pulse energy is 1.7 pJ. In Fig. 2(b), a similar result is presented for the TM gain. The results show that the largest decrease in the TE gain takes place if the pump pulse is also purely TE polarized and the smallest variation in the TE gain takes place if the pump pulse is purely TM polarized. It can be noted from Fig. 2 that there is a clear gain compression induced by the strong pump pulses. After its compression, an initial gain recovery at a 0.5 -ps timescale appears, then followed by a slow recovery time, which is associated with the interband effects determined by the electron and hole recombination times ( $1.3 \mathrm{~ns}$ in our cases, not shown in Fig. 2).

If the probe pulse is linearly polarized under an angle of $45^{\circ}$ with respect to the TE and TM polarization axes, we can compare simultaneously the phase change for both of its TE and TM polarization components. The pump-induced phase shifts between the TE and TM components of the probe pulse as a 


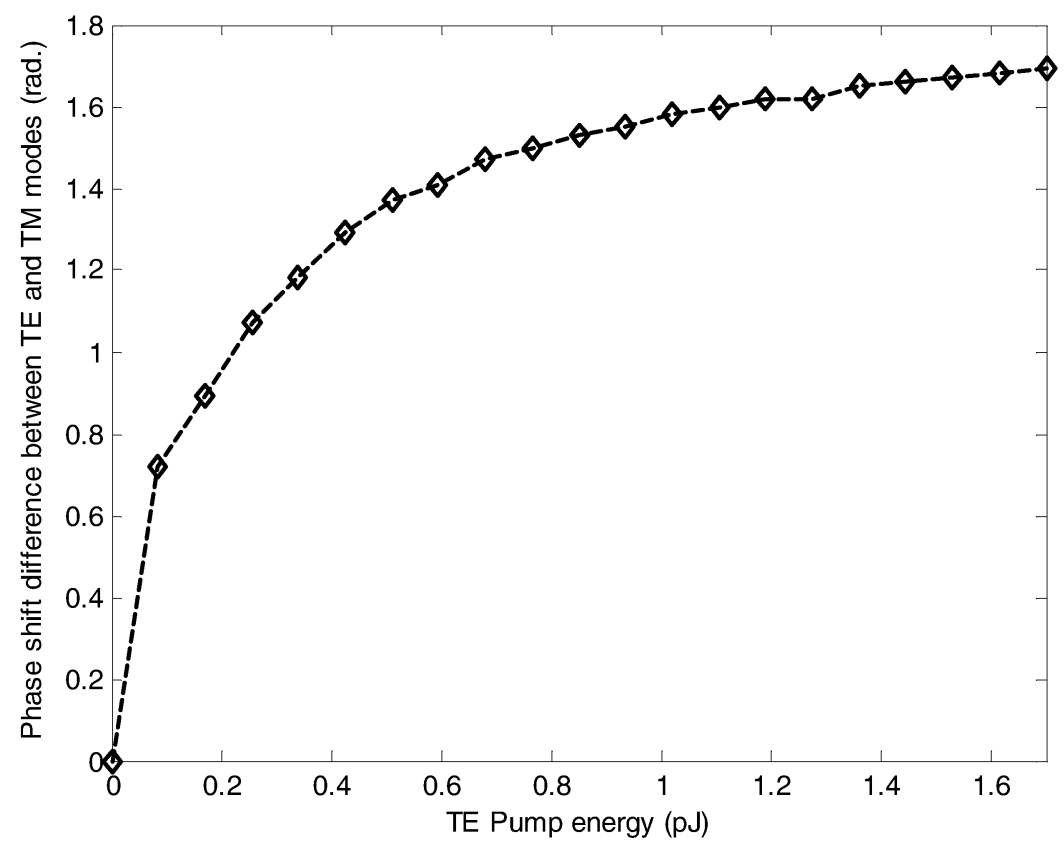

Fig. 3. Net phase shift difference $\Delta \phi$ between TE and TM modes $\left(\Delta \phi=\phi^{\mathrm{TE}}-\phi^{\mathrm{TM}}\right)$ as function of the energy of a TE polarized pump pulse.

function of the pulse energy of a TE polarized pump pulse is shown in Fig. 3. The results are calibrated with respect to the constant phase shift due to the intrinsic birefringence of the SOA when the current is $120 \mathrm{~mA}$. The largest pump-induced phase shift of a TE polarized probe pulse is about 1.8 radians for all pump polarizations at pulse energy of $1.7 \mathrm{pJ}$.

\section{SUbPICOSECOND NONLINEAR REFRACTIVE INDEX DYNAMICS}

As a first experiment, we wish to investigate the nonlinear phase shift introduced by ultrashort optical pulses in an SOA. The experimental method we used is referred to as spectral interferometry [62]. A sequence of two optical pulses, a probe pulse and a reference pulse, with well-defined phases enter the SOA. The pump-induced refractive-index change causes a phase shift of the probe pulse that is measured by interfering the probe pulse with the reference pulse. Measuring the probe phase shift as a function of the pump-probe delay enables the time-resolved measurement of ultrafast refractive-index changes. The optical spectrum of the probe and reference pulses has a modulation that is proportional to $\cos \left[\left(\omega-\omega_{0}\right) T+\phi\right]$, where $\omega$ is the optical frequency and $\omega_{0}$ is the central optical frequency of the pulses [62]. $T$ is the time between the pulses and $\phi$ is their relative phase. The modulation of the spectrum is inversely proportional to the time between the pulses and the modulation depth is proportional to the amplitude difference of the pulses [62]. The relative phase determines the positions of the minima and maxima of the fringes in spectrum. The (low-intensity) reference pulse is fully amplified, since the SOA is in equilibrium, but the (high-intensity) pump pulse is timed in such a way that it arrives at the SOA just before the probe pulse. Thus, the probe pulse will receive less amplification and undergo a phase shift, which is different from the reference pulse due to the gain saturation introduced by the pump pulse [63].
Fig. 4 is a schematic of the experimental setup. An optical parametric oscillator (OPO) pumped with a mode-locked Ti : Sapphire laser is used to produce optical pulses that were 140 fs (at FWHM) in duration and at a repetition rate of $80 \mathrm{MHz}$. The central wavelength of the pulses was $1550 \mathrm{~nm}$. The OPO output power is divided by a half mirror into two parts. The first half of the optical power is collimated into a single-mode optical fiber after passing through a polarization controller and an attenuator. A variable attenuator is employed to precisely control the pulse power. A Mach-Zehnder interferometer with unequal arms is utilized as fiber delay system to create the probe and reference pulse. The reference pulse is advancing the probe pulse by 100.4 ps. The second half of the OPO output forms the pump pulse. The pump pulse is subsequently sent through a polarization controller, an attenuator, and a variable delay line, and finally also fed into a single-mode optical fiber. The pump pulse and the probe and reference pulses are cross-linearly polarized to distinguish the pump light from the probe and reference pulse. The pulses are then combined by using an optical coupler and fed into the SOA by using a set of graded-index lenses. In the fiber system, the pulses are broadened to 300 fs due to dispersion. The total coupling losses are estimated to be $10 \mathrm{~dB}$. Measured at the coupler, the optical power of the pump signal was $705 \mu \mathrm{W}$ while the optical power of the combined probe and reference pulses was $61 \mu \mathrm{W}$. The SOA output is fed into an inline polarizer after passing through an isolator, an optical bandpass filter $(5 \mathrm{~nm})$ and a polarization controller. The inline polarizer is used to separate the pump light from the probe and reference pulse. After passing through the inline polarizer, the power ratio between the pump pulse, and the probe and reference pulse was $1: 14$. Finally, the optical spectrum of the probe and reference pulses are analyzed by using an optical spectrum analyzer with a resolution of $0.01 \mathrm{~nm}$. The relative phase is stable over several minutes without using active control, since the interferometer 


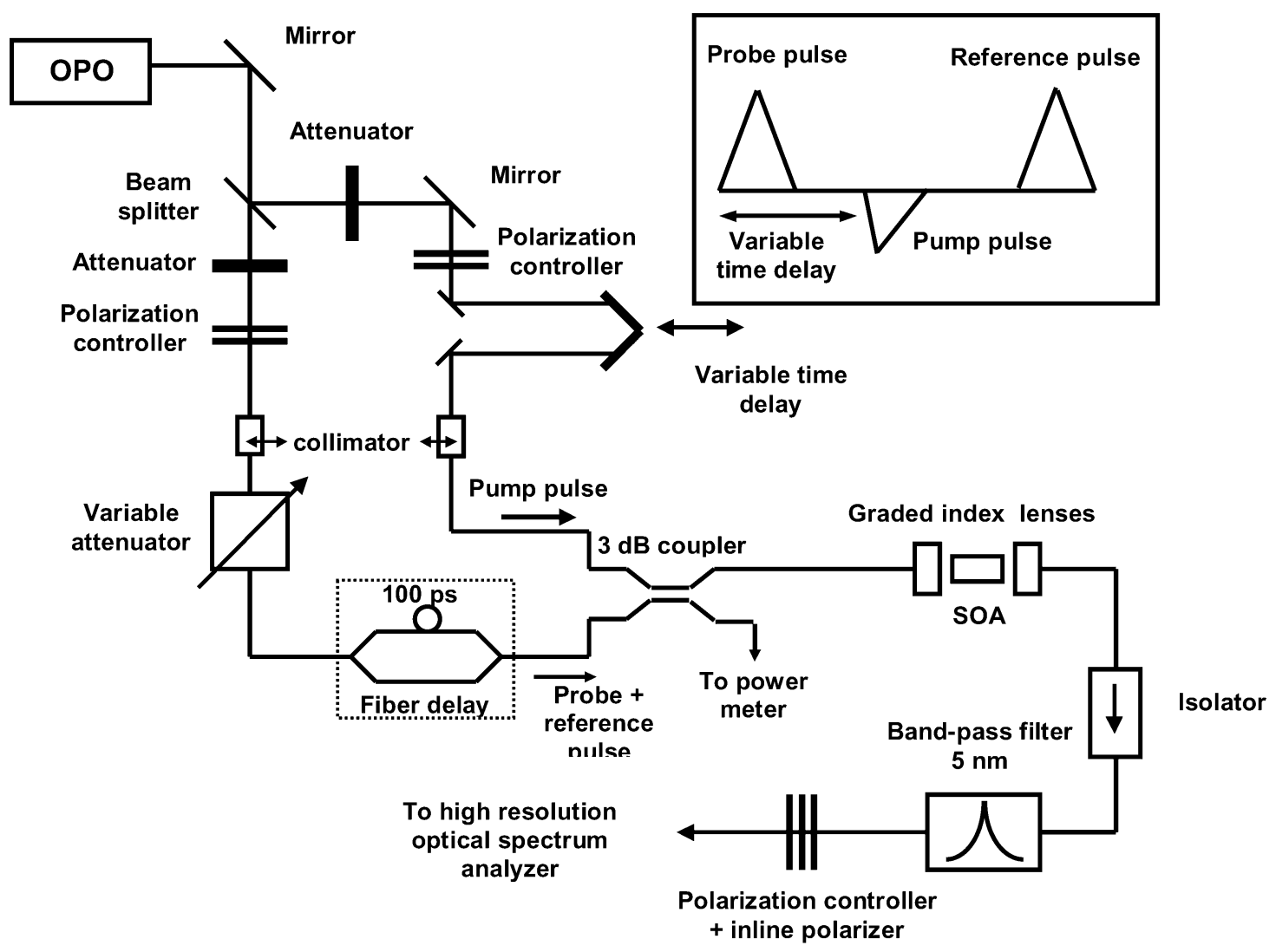

Fig. 4. Schematic of the experimental setup that is used to measure subpicosecond phase changes.

is made of fiber couplers and shielded in a box from thermal and mechanical disturbances.

In the first experiment, the SOA is pumped with $120 \mathrm{~mA}$ of current. Fig. 5 shows three traces of measured optical spectra of the probe and reference pulse in the presence and absence of pump light. The upper trace in series "A" is the optical spectrum of the probe and reference pulses in absence of pump light. The lower trace in series " $\mathrm{A}$ " is the measured optical spectrum if the pump pulse arrives after the probe and reference pulse. Consequently, the pump light does not affect the probe and reference pulses and, thus, no phase changes are visible. The two traces in series "B" are the optical spectra in the case that the pump pulse arrives at the SOA simultaneously with the probe pulse. The upper trace in series " $\mathrm{B}$ " is the spectrum of the probe and reference pulses in absence of pump light. Firstly, a decrease of the modulation depth of the spectrum is visible. This is due to the reduced amplification of the probe pulse in the presence of the pump light [62]. It is estimated that the probe pulse has received $2.7 \mathrm{~dB}$ less amplification compared to the reference pulse. Second, a phase shift of approximately $200^{\circ}$ is visible. This is due to the refractive index change introduced by the pump pulse. The two traces in series "C" represent the case that the pump pulse arrives $1.3 \mathrm{ps}$ before the probe pulse at the SOA. Again, the upper trace in series " $\mathrm{C}$ " is the spectrum of the probe and reference pulses in absence of pump light. From the reduced modulation depth, it can be concluded that the probe pulse has received less gain, but no phase difference is visible. Fig. 6 shows the phase change as a function of the pump-probe delay. It follows from Fig. 6 that the phase change recovers within a picosecond. In contrast to results for an InGaAsP-InP bulk SOA that are published in [63], we do not observe phase recovery effects on a timescale of a few picoseconds that can be associated with carrier heating. This might be related to the fact that we use an MQW SOA that has a smaller linewidth enhancement factor compared to a bulk SOA, in combination with our experimental approach; we measure the pump induced nonlinear phase with respect to a reference pulse that might create a long-lived tail by itself. In the second experiment, the SOA current was increased to $160 \mathrm{~mA}$ while all the other conditions were kept the same. The dashed line in Fig. 6 shows a vanishing phase change as a function of the pump-probe delay for $I=160 \mathrm{~mA}$. However, the reduced modulation depth of the optical spectrum reveals a maximum difference in amplification between the reference pulse and the probe pulse of $3.2 \mathrm{~dB}$.

Our results concerning the ultrafast phase change in the SOA can be explained by using the formula [55]

$$
\frac{\partial \phi}{\partial z}=\frac{1}{2} \alpha g-\frac{1}{2} \beta \alpha_{2} S
$$

where $\alpha$ is the linewidth enhancement factor, $g$ is the SOA gain, $\beta$ the TPA parameter, $\alpha_{2}$ the linewidth enhancement factor due to TPA, and $S$ the photon number, i.e., proportional to the optical pulse energy. The first term on the right-hand side of (18) describes the phase change due to the SOA gain that depends on the injection current. The second term on the right-hand side describes the phase change due to TPA. In the presence of a pump pulse, the first term will always lead to a negative phase shift contribution with respect to the situation without pump 


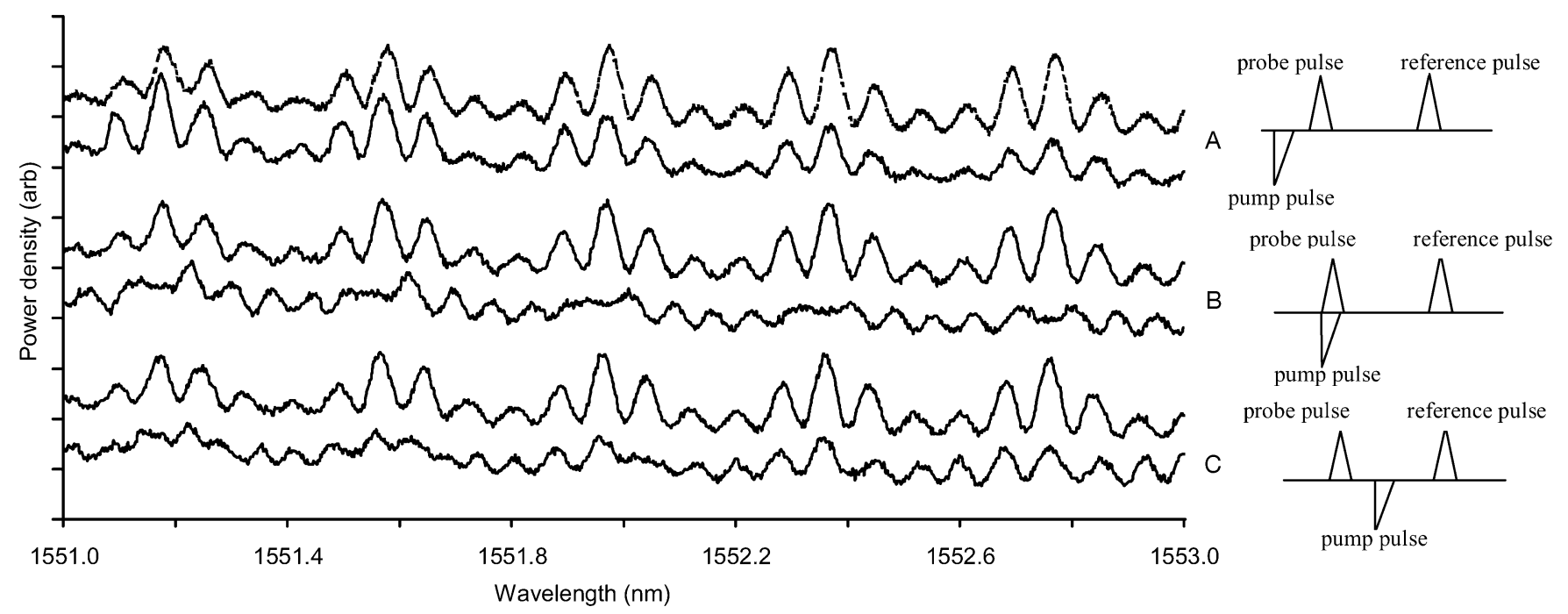

Fig. 5. Optical spectra of a probe and reference pulse in the case that the pump pulse: (A) arrives after the probe and reference pulse; (B) arrives almost simultaneously with the probe pulse; and (C) arrives 1.3 ps before the probe pulse. The injection current is $120 \mathrm{~mA}$. The upper trace in (A), (B), and (C) represents the interference spectrum in absence of pump light. The $50-\mathrm{GHz}$ modulation that is visible in the spectra is an instrumental artifact related to "aliasing."

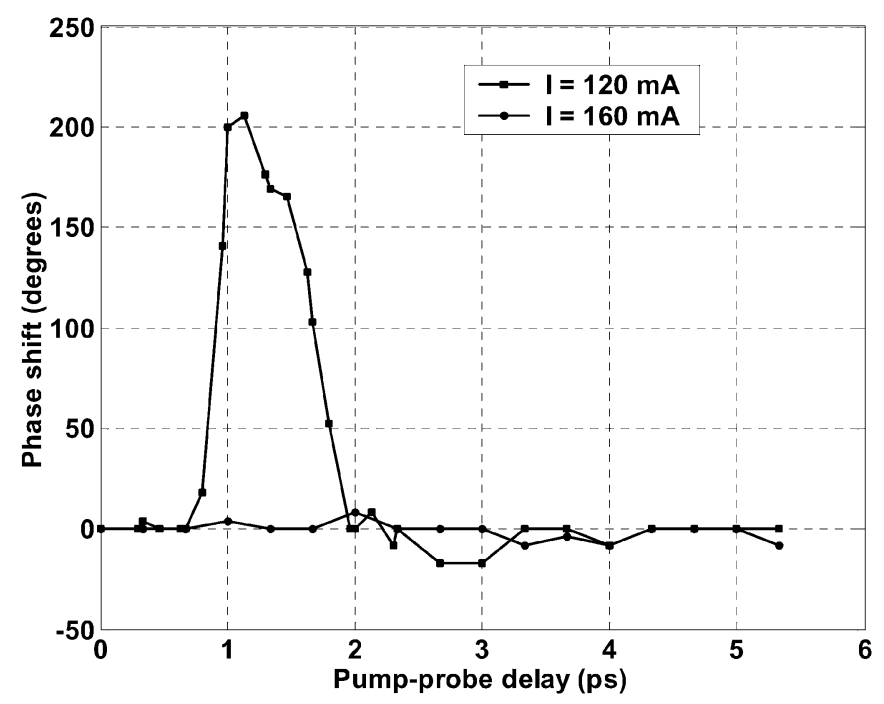

Fig. 6. Phase change as a function of the pump-probe delay for injection currents of $120 \mathrm{~mA}$ (solid line) and $160 \mathrm{~mA}$ (dashed line). The error margin is $10^{\circ}$.

pulse. This contribution increases in magnitude with increasing bias current. The second term is only nonzero in the presence of a pump pulse. Clearly, the observed compensating effect for higher current can only be explained when $\alpha_{2}<0$.

\section{Ultrafast All-Optical Switch}

The results shown in Section IV show that ultrashort optical pulses can introduce nonlinear phase changes larger than $180^{\circ}$ in an SOA. In this section, we investigate an optical switch based on ultrafast SOA index nonlinearities.

A schematic of our all-optical logic AND gate is shown in Fig. 7. An OPO pumped with a mode-locked Ti : Sapphire laser is used to produce optical pulses that were $200 \mathrm{fs}(\mathrm{FWHM})$ in duration at a repetition rate of $75 \mathrm{MHz}$. The central wavelength

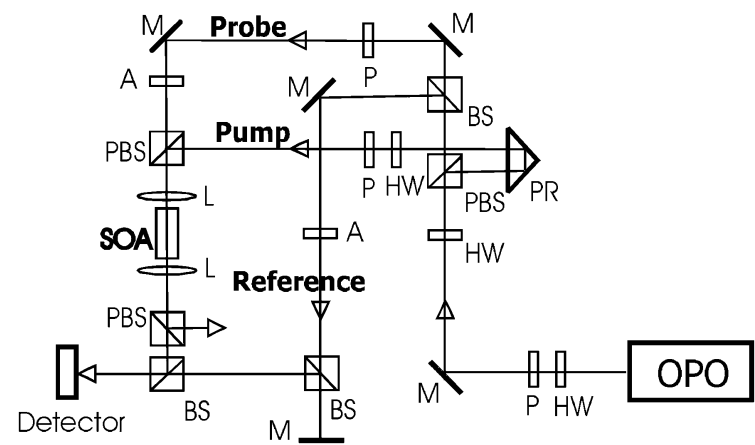

Fig. 7. Schematic of the experimental setup, where the symbols of optical components are defined as follows: OPO: optical parametric oscillator, HW: half-wave plate, $\mathrm{P}$ is polarizer, M: mirror, PBS: polarizing beam splitter, BS: beam splitter, PR: prism, A: attenuator, L: lens.

of the pulses was $1520 \mathrm{~nm}$. The OPO output is firstly attenuated using a half-wave plate and a polarizer. A second half-wave plate is used to set the polarization of the laser beam to linear under $45^{\circ}$. A polarizing beam splitter is used to create a TE polarized laser beam and a TM polarized laser beam. The TM polarized laser beam forms the pump light and is fed into a variable delay line. A beam splitter divides the TE polarized laser beam into a probe beam and a reference beam. The pump and probe beams are coupled into the SOA through microscope objectives. The coupling losses are estimated to be $6 \mathrm{~dB}$. The pump-probe delay is controlled by the variable delay stage. The SOA used in our experiments is an InGaAsP-InGaAs MQW SOA with a central length of $750 \mu \mathrm{m}$ and a taper zone with a length of $400 \mu \mathrm{m}$ on each sides of the central part. Neutral density filters are used to control the power of the probe and reference beams. A translatable end mirror controls the delay between the reference beam and the probe beam. When the pump and probe beams have passed through the SOA, the pump light is removed by using another polarizing beam splitter. The probe and reference beams first interfere in the beam splitter. The interfered light is then collimated into an optical fiber that is connected to a detector. The whole setup is placed in a polystyrene 


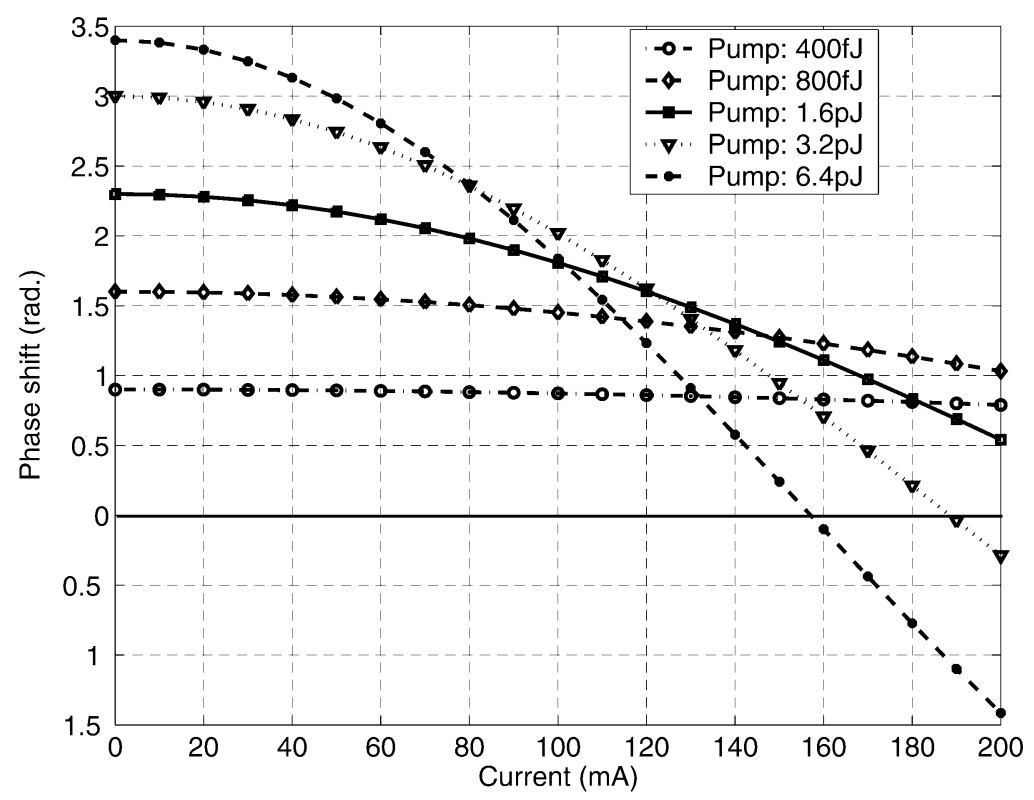

Fig. 8. Nonlinear phase shifts as a function of the injection current for various pump and probe energies when the pump-probe delay is zero.

housing box to shield the interferometer from thermal and mechanical disturbances. This makes it so that the relative phase is stable over several minutes without any active control. An automated measurement system is employed to obtain stable measurements without any active control.

In our first experiment, the interferometer output power is measured for zero pump-probe delay. The power of the probe beam was equal to the power of the reference beam, but the pump power was ten times larger. We have observed that our SOA converts about $1 \%$ of the TM polarized pump light into TE polarized light, which also interferes with the probe and reference beams. First, we measured the maximum interferometer output (i.e., with zero time delay between probe and reference pulse) as a function of the injection current. The interferometer output power can be related to the nonlinear phase shift through the following relationship:

$$
\begin{aligned}
S_{\mathrm{det}}(\tau)=\frac{1}{T} \int_{-T / 2}^{T / 2}\{ & P_{p}(t+\tau)+P_{\mathrm{pr}}(t)+P_{r}(t) \\
& +2 \sqrt{P_{r}(t) P_{p}(t)} \cos \left[\phi_{r, p}-a I\right] \\
& +2 \sqrt{P_{p}(t+\tau) P_{\mathrm{pr}}(t)} \\
& \times \cos \left[\phi_{p, \mathrm{pr}}-\Delta \phi_{\mathrm{NL}}(\tau)\right] \\
& +2 \sqrt{P_{r}(t) P_{\mathrm{pr}}(t)} \\
& \left.\times \cos \left[a I+\Delta \phi_{\mathrm{NL}}(\tau)\right]\right\} d t
\end{aligned}
$$

where $S_{\text {det }}(\tau)$ is the detector signal, $\tau$ the pump-probe delay, $P_{p}(t+\tau)$ is the power of the polarization converted pump pulse, $P_{\mathrm{pr}}(t)$ is the power of the probe pulse and $P_{r}(\tau)$ is the power of the reference pulse. The pump-induced nonlinear phase shift $\Delta \phi_{\mathrm{NL}}(\tau)$ is the phase difference of the probe pulse between the cases in presence and absence of a pump pulse. $\phi_{r, p}$ is the phase shift introduced by the optical path length difference between the reference pulse and the polarization converted pump pulse. Similarly, $\phi_{p, \text { pr }}$ represents the phase shift introduced by the path length difference between the polarization converted pump pulse and the probe. Finally, we have observed that the phase shift induced by the SOA injection current $I$ equals to $a I$, where $a=\pi / 100 \mathrm{rad} / \mathrm{mA}$. The detector response time $T$ in (19) is much larger than the pulse duration. Fig. 8 shows the measured $\Delta \phi_{\mathrm{NL}}(\tau)$ as a function of the injection current. It follows that for small injection currents, large positive phase shifts can be obtained. These results are in qualitative agreement with the results published in [64], where it is shown that the phase shift due to TPA opposes the phase shift introduced by the gain.

The nonlinear phase shift per SOA unit length can be expressed as $2\left(\partial \Delta \phi_{\mathrm{NL}}(\tau) / \partial z\right)=\alpha\left[g_{p}(\tau)-g_{0}\right]-\alpha_{2} \beta_{2} S(\tau)$ [64]. Here, $\alpha$ is the linewidth enhancement factor, $\beta_{2}$ the TPA coefficient, $\alpha_{2}$ the linewidth enhancement factor associated with TPA, $S(\tau)$ the photon number of the injected light, $g_{p}(\tau)$ the gain in presence of a pump pulse, and $g_{0}$ is the gain in absence of the pump light. The gain difference in the first contribution describes the well-known gain depletion and ultrafast recovery due to carrier cooling versus pump-probe delay time [37]. This term has an overall proportionality to the injection current $I$. For sufficiently small currents, the first contribution is positive due to dominating absorption. For a certain current, which depends on the pump pulse energy, this term turns negative due to depletion. The second term is proportional to, and has the same shape as, the pump pulse. It was already concluded in the previous section that $\alpha_{2}$ has a negative sign. Therefore, the contribution of the second term to the phase shift is always positive. Hence, at zero current we predict the highest phase shifts, while for higher currents the phase shift decreases due to the smaller contribution of the first term.

If an ultrashort optical pump pulse is fed into an SOA that is operated at zero injection current, it will generate carriers, not only directly by absorption but also by TPA. These latter carriers are hot, but will cool down on a subpicosecond timescale and will already lead to an extra increase of the gain within the 


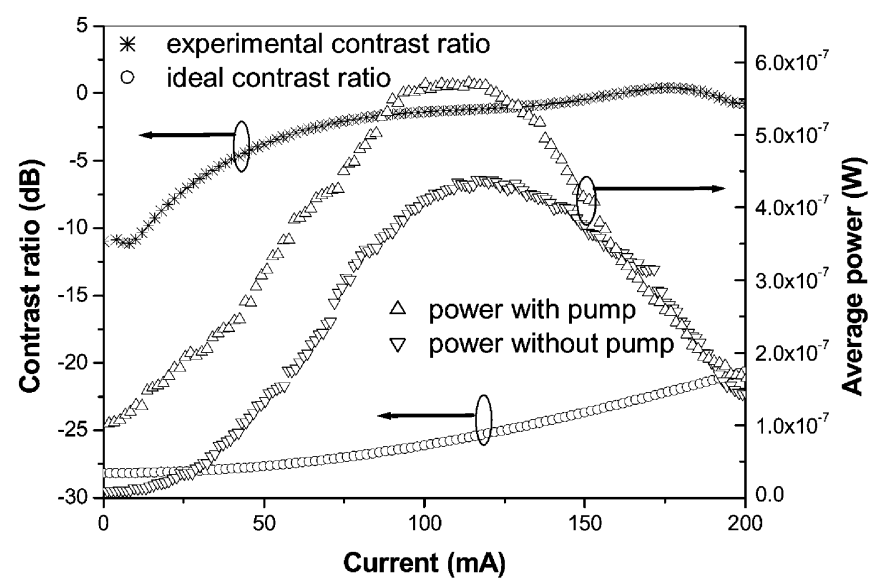

Fig. 9. Average output powers of the AND gate as a function of the injection current in presence and absence of pump light. Also shown is the contrast ratio. The pump pulse energy is $800 \mathrm{fJ}$ while the probe (or reference) pulse energy is $80 \mathrm{fJ}$. The "ideal" curve shows the calculated contrast ratio that could be achieved if the polarization converted pump pulse residue could be removed.

carrier-carrier scattering time (50-100 fs), all of which leads to a positive phase shift. On the other hand, if the injection current is increased to a value above transparency, a reservoir of carriers is available in the conduction and valence bands. As soon as the optical pump pulse passes by, these carriers recombine due to stimulated emission followed by a recovery of the carrier number due to TPA and cooling. In this case, the negative gain induced contribution to the phase shift counteracts the positive instantaneous TPA induced contribution. For a specific injection current, the value of which depends on the pump pulse energy, the net phase shift vanishes. For this injection current, the phase shift due to the stimulated emission is precisely compensated by the phase shift due to TPA [64]. If the injection current is increased further, the net phase shift is dominated by the stimulated emission and saturates for high injection currents.

We have also measured the contrast ratio of the optical gate using pump pulses of 800-fJ energy. The result is shown in Fig. 9, where also the output power of the gate is plotted in the presence and absence of a pump pulse. According to Fig. 9, the best result with 800-fJ pump pulses is expected for $I=0$. Therefore, the setup was calibrated at $I=0$ to achieve a minimum output without pump light. We measure for $I=0$ a contrast ratio of $-11 \mathrm{~dB}$. This value is limited by the residual noise in the output without pump pulse, as well as by the polarization converted pump pulse residue as discussed below (19). It can be derived from (19) that given the observed noise level, if the pump pulse residue could be suppressed, the ideal contrast ratio can be as small as $-26 \mathrm{~dB}$, increasing slowly with $I$. This is indicated by the curve labeled "ideal" in Fig. 9. The ideal curve is computed by putting $P_{p}(t)$ to zero in (19), while keeping all the other values the same. In this latter curve, it is assumed that for each value of $I$, the system is calibrated to the minimum noise floor level in the output without pump pulse (which has not been done in the experiment). The contrast ratio can be further improved by optimizing the delay between the pump and probe pulses, the probe and reference pulse, as well as by lowering the noise floor in the output without pump pulse. In addition, we observed from the optical spectra that the output pulse did not significantly broaden after passing through the gate.

\section{DISCUSSION}

We have presented in this paper an optical logic AND gate which operated by ultrafast carrier dynamics in an SOA. Operation has been demonstrated by using ultrashort pulses at a low repetition rate. We have also presented a rate-equation model that describes carrier dynamics in the SOA at subpicosecond timescales.

The largest technological challenge on the road toward the implementation of ultrafast optical logic based on SOA nonlinearities operated at ultrahigh repetition rates is undoubtedly related to the power consumption of these devices. Fig. 1 shows that an optical pulse with energy of $200 \mathrm{fJ}$ can suppress the SOA amplification with approximately $15 \mathrm{~dB}$. This implies that at a bit rate of $1 \mathrm{~Tb} / \mathrm{sec}$ an average power of at least $100 \mathrm{~mW}$ is required to introduce nonlinearities that are strong enough to allow bit-wise optical switching (here it is assumed that half of the transmitted data are zeros). If switching principles based on nonlinear refractive index dynamics are employed, Fig. 9 shows that pulse energies in the order of $3 \mathrm{pJ}$ are required to introduce a phase shift of $\pi$ radians. As shown in Section V, switching can be realized using pulses with much lower energy, at the expense of a high extinction ratio. In Section V, we obtained an extinction ratio of $11 \mathrm{~dB}$ using control pulses with an energy of $800 \mathrm{fJ}$ in an asymmetric Mach-Zehnder interferometer. Commercially available SOAs can handle average input powers with a maximum of $50 \mathrm{~mW}$, which implies that the SOA nonlinearities are too weak to allow bitwise optical switching at ultrahigh repetition rates. We wish to remark that the coupling losses presented in this paper have been estimated conservatively, thus we expect that in reality the switching powers will be lower.

In [57], results are published showing that in a $250-\mu \mathrm{m}$-long InGaAsp-InP bulk SOA, negative gain values are reached for pulses with energy of $100 \mathrm{fJ}$ [57]. Pump and probe studies on the same device reveal that pulse energies between $460 \mathrm{fJ}$ and a few picojoules (depending on whether the SOA is in the gain or absorption regime) are required to obtain phase shifts larger than $2 \mathrm{rad}$ [63]. In our device, optical pulses with energy of $800 \mathrm{fJ}$ can introduce a nonlinear phase shift of $2.3 \mathrm{rad}$.

The observed switching energies in SOAs is relatively low compared to the switching energies in passive intersubband waveguides in which switching energies in the order of $10 \mathrm{pJ}$ per pulse are required to create an extinction ratio of $10 \mathrm{~dB}$ [65]. Also, the powers required to obtain switching in an SOA seem to be an order lower compared to electroabsorption modulators [2], [66]. Note that Fig. 9 shows that the largest nonlinear phase shifts in our SOA are obtained at zero injection current. This result also suggests that it is beneficial to carry out optical time domain multiplexing (OTDM) demuliplexing at ultrahigh bit rate by using passive devices.

A second challenge is to employ ultrafast SOA nonlinearities in more sophisticated ultrafast all-optical logic circuits. The main hurdle to be taken here is also related to the power consumption of the circuit. More complex optical logic functionalities such as optical flip-flop memories or optical pseudorandom number generators are often realized by coupling two all-optical logic gates so that the output signal of the first logic gate acts as a control signal for the second logic gate [23], [27]. The 
optical logic gates are usually implemented by employing SOA refractive index nonlinearities in an interferometric environment (usually SOAs in a Sagnac interferometer or a Mach-Zehnder interferometer). It follows from Fig. 1 that if pulses with pulse energies greater than $2 \mathrm{pJ}$ are injected in our SOA, the output pulse energies are smaller than the input pulse energies. Fig. 9 reveals that pulse with energies of $2 \mathrm{pJ}$ can introduce a nonlinear phase shift of approximately $2.7 \mathrm{rad}$. If nonlinear phase shifts greater than 2.7 rad are required, the output pulse energy of the first gate will be insufficient to control the second gate. Sophisticated integrated optical circuits consist of active elements coupled to each other through passive elements such as splitters and filters which introduce additional losses [67]. It is, therefore, essential to design all-optical logic circuits that can be controlled with pulses with energies far below the critical $2 \mathrm{pJ}$ limit.

It might be possible to design optical logic gates that can be controlled with pulses of much lower energies. Interesting and promising examples include differentially operated Mach-Zehnder interferometers or nonlinear polarization switches [46], [68]. It is also believed that lower power operation can be obtained by employing quantum dot SOA. Recently the first prototype quantum dot SOAs operating at telecommunication wavelengths have been realized [69]. It should be realized however that the nonlinear carrier dynamics of quantum dot SOAs differs fundamentally from the results presented in this paper [70], and it is not yet clear how quantum dot devices can be employed in optical logic.

\section{REFERENCES}

[1] M. Nakazawa, T. Yamamoto, and K. Tamura, "12.8 Tbit/s-70 km OTDM transmission using third- and fourth order simultaneous dispersion compensation with a phase modulator," Electron. Lett., vol. 36, pp. 2027-2029, 2000.

[2] D. Cotter, R. J. Manning, K. J. Blow, A. D. Ellis, A. E. Kelly, D. Nesset, I. D. Phillips, A. J. Poustie, and D. C. Rogers, "Nonlinear optics for highspeed digital information processing," Science, vol. 286, pp. 1523-1528, 1999.

[3] J. P. Sokoloff, P. R. Prucnal, I. Glesk, and M. Kane, "A terahertz optical asymmetric demultiplexer (TOAD)," IEEE Photon. Technol. Lett., vol. 5, pp. 787-790, July 1993.

[4] D. M. Patrick, A. D. Ellis, D. A. O. Davies, M. C. Tatham, and G. Sherlock, "Demultiplexing using polarization rotation in a semiconductor laser amplifier," Electron. Lett., vol. 30, pp. 341-342 , 1994.

[5] E. Jahn, N. Agrawal, M. Arbert, H. J. Ehrke, D. Franke, R. Ludwig, W. Pieper, H. G. Weber, and C. M. Weinert, " $40 \mathrm{Gbit} / \mathrm{s}$ all-optical demultiplexing using a monolithically integrated Mach-Zehnder interferometer with semiconductor laser amplifier," Electron. Lett., vol. 31, pp. 1857-1858, 1995.

[6] K. Tajima, S. Nakamura, and Y. Sugimoto, "Ultrafast polarization-discriminating Mach-Zehnder all-optical switch," Appl. Phys. Lett., vol. 67, pp. 3709-3711, 1995.

[7] N. S. Patel, K. L. Hall, and K. A. Rauschenbach, " 40 Gbit/sec cascadable all-optical logic with an ultrafast nonlinear interferometer," Opt. Lett., vol. 21, pp. 1466-1468, 1996.

[8] M. Eiselt, W. Pieper, and H. G. Weber, "SLALOM: semiconductor laser amplifier in a loop mirror," J. Lightwave Technol., vol. 13, pp. 2099-2112, Oct. 1995.

[9] R. J. Manning and D. A. O. Davies, "Three wavelength device for alloptical signal processing," Opt. Lett., vol. 28, pp. 1505-1507, 1994.

[10] M. T. Hill, E. Tangdiongga, H. de Waardt, G. D. Khoe, and H. J. S. Dorren, "Carrier recovery times in semiconductor optical amplifiers that employ holding beams," Opt. Lett., vol. 27, pp. 1625-1627, 2002.

[11] M. Sheik-Bahae, D. C. Hutchings, D. J. Hagan, and E. W. van Stryland, "Dispersion of bound electron nonlinear refraction in solids," IEEE $J$. Quantum Electron., vol. 27, pp. 1296-1309, June 1991.
[12] C. T. Hultgren, D. J. Dougherty, and E. P. Ippen, "Above- and belowband femtosecond nonlinearities in active AlGaAs waveguides," Appl. Phys. Lett., vol. 61, pp. 2767-2769, 1992.

[13] I. Glesk, K. I. Kang, and P. R. Prucnal, "All-optical address recognition and self-routing in a $250 \mathrm{Gbit} / \mathrm{s}$ packet switched," Electron. Lett., vol. 30, pp. 1322-1323, 1994.

[14] D. Cotter, J. K. Lucek, M. Shabeer, K. Smith, D. C. Rogers, D. Nesset, and P. Gunning, "Self routing of $100 \mathrm{Gbit} / \mathrm{sec}$ packets using 6 bit 'keyword' address recognition," Electron. Lett., vol. 31, pp. 1475-1476, 1995.

[15] N. Calabretta, H. de Waardt, G. D. Khoe, and H. J. S. Dorren, "Ultrafast asynchronous multioutput all-optical header processor," IEEE Photon. Technol. Lett., vol. 16, pp. 1182-1184, Apr. 2004.

[16] T. J. Xia, Y. H. Kao, Y. Liang, J. W. Lou, K. H. Ahn, O. Boyraz, G. A. Nowak, A. A. Said, and M. N. Islam, "Novel self-synchronization scheme for high-speed packet TDM networks," IEEE Photon. Technol. Lett., vol. 11, pp. 269-271, Feb. 1999.

[17] N. Calabretta, Y. Liu, F. M. Huijskens, M. T. Hill, H. de Waardt, G. D. Khoe, and H. J. S. Dorren, "Optical signal processing based on selfinduced polarization rotation in a semiconductor optical amplifier," $J$. Lightwave Technol., vol. 22, pp. 372-381, Feb. 2004.

[18] O. Kamatani and S. Kawanishi, "Ultrahigh-speed clock recovery with phase lock loop based on four wave mixing in a travelling wave laser diode amplifier," J. Lightwave Technol., vol. 14, pp. 1757-1767, Aug. 1996.

[19] A. J. Poustie, R. J. Manning, and K. J. Blow, "All-optical circulating shift register using a semiconductor optical amplifier in a fiber loop mirror," Electron. Lett., vol. 32, pp. 1215-1216, 1996.

[20] K. L. Hall, J. P. Donnely, S. H. Groves, C. I. Fennely, R. J. Bailey, and A. Napoleone, " $40 \mathrm{Gbit} / \mathrm{sec}$ all-optical circulating shift register with an inverter," Opt. Lett., vol. 22, pp. 1479-1481, 1997.

[21] A. J. Poustie, K. J. Blow, and R. J. Manning, "All-optical regenerative memory for long term data storage threshold and amplitude," Opt. Commun., vol. 140, pp. 184-186, 1997.

[22] — - "Storage threshold and amplitude restoration in optical regenerative memory," Opt. Commun., vol. 146, pp. 262-267, 1998.

[23] M. T. Hill, H. de Waardt, G. D. Khoe, and H. J. S. Dorren, "Fast optical flip-flop by use of Mach-Zehnder interferometers," Microw. Opt. Technol. Lett., vol. 31, pp. 411-415, 2001.

[24] H. J. S. Dorren, D. Lenstra, Y. Liu, M. T. Hill, and G. D. Khoe, "Nonlinear polarization rotation in semiconductor optical amplifiers: Theory and application to all-optical flip-flop memories," IEEE J. Quantum Electron., vol. 39, pp. 141-147, Jan. 2003.

[25] A. J. Poustie, K. J. Blow, A. E. Kelly, and R. J. Manning, "All-optical binary half-adder," Opt. Commun., vol. 156, pp. 22-26, 1998.

[26] - "All-optical full-adder with bit differential delay," Opt. Commun., vol. 168, pp. 89-93, 1999.

[27] A. J. Poustie, K. J. Blow, R. J. Manning, and A. E. Kelly, "All-optical pseudorandom number generator," Opt. Commun., vol. 159, pp. 208-214, 1999.

[28] A. J. Poustie, K. J. Blow, A. E. Kelly, and R. J. Manning, "All-optical parity checker with bit-differential delay," Opt. Commun., vol. 146, pp. 37-43, 1999.

[29] M. T. Hill, A. Srivatsa, N. Calabretta, Y. Liu, H. de Waardt, G. D. Khoe, and H. J. S. Dorren, " $1 \times 2$ all-optical packet switch using all-optical header processing," Electron. Lett., vol. 37, pp. 774-775, 2001.

[30] K. L. Hall, G. Lenz, A. M. Darwish, and E. P. Ippen, "Subpicosecond gain and index nonlinearities in InGaAsP diode lasers," Opt. Commun., vol. 111, pp. 589-612, 1994.

[31] M. P. Kessler and E. P. Ippen, "Subpicosecond gain dynamics in GaAlAs laser diodes," Appl. Phys. Lett., vol. 51, pp. 1765-1767, 1987.

[32] K. L. Hall, J. Mark, E. P. Ippen, and G. Eisenstein, "Femtosecond gain dynamics in InGaAsP optical amplifiers," Appl. Phys. Lett., vol. 56, pp. 1740-1742, 1990.

[33] K. L. Hall, E. P. Ippen, and G. Eisenstein, "Bias-lead monitoring of ultrafast nonlinearities in InGaAsP," Appl. Phys. Lett., vol. 57, pp. 129-131, 1990.

[34] K. L. Hall, G. Lenz, E. P. Ippen, and G. Raybon, "Heterodyne pump-probe technique for time-domain studies of optical nonlinearities in waveguides," Opt. Lett., vol. 17, pp. 874-876, 1992.

[35] B. N. Gomatam and A. P. DeFonso, "Theory of hot carrier effects on nonlinear gain in GaAs-GaAlAs lasers and amplifiers," IEEE J. Quantum Electron., vol. 26, pp. 1689-1704, Oct. 1990.

[36] J. Mark and J. Mørk, "Sub-picosecond gain dynamics in InGaAsP optical amplifiers, experiment and theory," Appl. Phys. Lett., vol. 61, pp. 2281-2283, 1992. 
[37] J. Mørk, J. Mark, and C. P. Seltzer, "Carrier heating in InGaAsP laser amplifiers due to two-photon absorption," Appl. Phys. Lett., vol. 64, pp. 2206-2208, 1994.

[38] K. L. Hall, G. Lenz, E. P. Ippen, U. Koren, and G. Raybon, "Carrier heating and spectral hole burning in strained-layer quantum well laser amplifiers at $1.5 \mu \mathrm{m}$," Appl. Phys. Lett., vol. 61, pp. 2512-2514, 1992.

[39] C. T. Hultgren and E. P. Ippen, "Ultrfast refractive index dynamics in AlGaAs diode laser amplifiers," Appl. Phys. Lett., vol. 59, pp. 635-637, 1991.

[40] K. L. Hall, Y. Lai, E. P. Ippen, G. Eisenstein, and U. Koren, "Femtosecond gain dynamics and saturation behavior of InGaAsP multiple quantum well optical amplifiers," Appl. Phys. Lett., vol. 57, pp. 2888-2890, 1990

[41] K. L. Hall, A. M. Darwish, E. P. Ippen, and G. Raybon, "Femtosecond index nonlinearities in InGaAsP optical amplifiers," Appl. Phys. Lett., vol. 62, pp. 1320-1322, 1993.

[42] J. Mørk, M. Willatzen, J. Mark, M. Svendsen, and C. P. Seltzer, "Characterization and modeling of ultrafast carrier dynamics in quantum well optical amplifiers," Proc. SPIE, Physics and Simulation of Optoelectronic Devices II , vol. 2146, pp. 52-67, 1994.

[43] A. Mecozzi and J. Mørk, "Theory of heterodyne pump-probe experiments with femtosecond pulses," J. Opt. Soc. Amer. B, vol. 13, pp. 2437-2452, 1996

[44] S. Hughes, A. Knorr, and S. W. Koch, "Interplay of optical dephasing anf pulse propagation in semiconductors," J. Opt. Soc. Amer., Opt. Phys., vol. 49, pp. 754-760, 1997.

[45] W. W. Chow and S. W. Koch, Semiconductor Laser Fundamentals. Berlin, Germany: Springer-Verlag, 1999

[46] S. Nakamura, Y. Ueno, K. Tajima, J. Sasaki, T. Sugimoto, T. Kato, T. Shimoda, M. Itoh, H. Hatakeyama, T. Tamanuki, and T. Sasaki, "Demultiplexing of $168-\mathrm{Gb} / \mathrm{s}$ data pulses with a hybrid-integrated symmetric Mach-Zehnder all-optical switch," IEEE Photon. Technol. Lett., vol. 12, pp. 425-427, Apr. 2000.

[47] S. Nakamura, Y. Ueno, and K. Tajima, "Femtosecond switching with semiconductor optical amplifier based symmetric Mach-Zehnder type optical switch," Appl. Phys. Lett., vol. 78, pp. 3929-3931, 2001.

[48] - "Ultrafast (200 fs, $1.5 \mathrm{~Tb} / \mathrm{s}$ demultiplexing) and high repetition $(10 \mathrm{Ghz})$ operations of a polarization discriminating symmetric Mach-Zehnder all-optical switch," IEEE Photon. Technol. Lett., vol. 10, pp. 1575-15477, Nov. 1998.

[49] H. Kuwatsuka, T. Akiyama, B. E. Little, T. Simoyama, and H. Ishikawa, "Wavelenght conversion of picosecond optical pulses using four wave mixing in a DFB laser," in Proc. Eur. Conf. Optical Communication 2000 , vol. 3, pp. 65-66.

[50] T. Akiyama, O. Wada, H. Kuwatsuka, T. Simoyama, Y. Nakata, K. Mukai, M. Sugawara, and H. Ishikawa, "Nonlinear processes responsible for nondegenerate four-wave mixing in quantum-dot optical amplifier," Appl. Phys. Lett., vol. 74, pp. 1753-1755, 2000.

[51] T. Akiyama, H. Kuwatsuka, N. Hatori, Y. Nakata, H. Ebe, and M. Sugawara, "Symmetric highly efficient $(0 \mathrm{~dB})$ wavelength conversion based on four-wave-mixing in quantum dot optical amplifiers," IEEE Photon. Technol. Lett., vol. 14, pp. 1139-1141, Aug. 2002.

[52] X. Yang, D. Lenstra, G. D. Khoe, and H. J. S. Dorren, "Nonlinear polarization rotation induced by ultrashort optical pulses in semiconductor optical amplifier," Opt. Commun., vol. 223, pp. 169-179, 2003.

[53] S. Bischoff, A. Buxens, S. Fisher, M. Dülk, A. T. Clausen, H. N. Poulsen, and J. Mørk, Opt. Quantum Electron., vol. 33, pp. 907-907, 2003.

[54] M. Y. Hong, Y. H. Chang, A. Dienes, J. P. Heritage, P. J. Delfyett, S. Dijaili, and F. G. Patterson, "Femtosecond self- and cross-phase modulation in semiconductor laser amplifiers," IEEE J. Select. Topics Quantum Electron., vol. 2, pp. 523-539, Sept. 1996.

[55] H. J. S. Dorren, G. D. Khoe, and D. Lenstra, "All-optical switching of an ultrashort pulse using a semiconductor optical amplifier in a Sagnacinterferometric arrangement," Opt. Commun., vol. 205, pp. 247-252, 2002.

[56] M. T. Hill, H. de Waardt, G. D. Khoe, and H. J. S. Dorren, "All-optical flip-flop based on coupled laser diodes," IEEE J. Quantum Electron., vol. 37, pp. 405-413, Mar. 2001.

[57] F. Romstad, P. Borri, W. Langbein, J. Mørk, and J. M. Hvam, "Measurement of pulse amplitude and phase distortion in a semiconductor optical amplifier: From pulse compression to breakup," IEEE Photon. Technol. Lett., vol. 12, pp. 1674-1676, Dec. 2000.

[58] T. D. Visser, H. Blok, and D. Lenstra, "Theory of polarization dependent amplification in a slab waveguide with anisotropic gain and losses," IEEE J. Quantum Electron., vol. 35, pp. 240-249, Feb. 1999.

[59] T. D. Visser, H. Blok, B. Demeulenaere, and D. Lenstra, "Confinement factors and gain in optical amplifiers," IEEE J. Quantum Electron., vol. 33, pp. 1763-1766, Oct. 1997
[60] B. M. Yu and J. M. Liu, "Polarization dependent gain, gain nonlinearities and emission characteristics of internally strained InGaAsP/InP semiconductor lasers," J. Appl. Phys., vol. 69, pp. 7444-7459, 1991.

[61] Y. Takahashi, A. Neogi, and H. Kawaguchi, "Polarization-dependent nonlinear gain in semiconductor lasers," IEEE J. Quantum Electron., vol. 34, pp. 1660-1672, Sept. 1998.

[62] A. P. Heberle, J. J. Baumberg, and K. Kohler, "Ultrafast coherent control and destruction of excitons in quantum wells," Phys. Rev. Lett., vol. 75, pp. 2598-2600, 1995.

[63] P. Borri, W. Langbein, J. Mørk, and J. M. Hvam, "Heterodyne pumpprobe and four-wave mixing in a semiconductor optical amplifier using balance lock-in detection," Opt. Commun., vol. 169, pp. 317-324, 1999.

[64] H. J. S. Dorren, X. Yang, D. Lenstra, H. de Waardt, G. D. Khoe, T Simoyama, H. Ishikawa, H. Kawashima, and T. Hasama, "Ultrafast refractive-index dynamics in a multiquantum-well semiconductor optical amplifier," IEEE Photon. Technol. Lett., vol. 15, pp. 792-794, June 2003.

[65] T. Simoyama, H. Yoshida, J. Kasai, T. Mozume, A. V. Gopal, and H. Ishikawa, "InGaAs-AlAs-AlAsSb coupled quantum well intersubband transition all-optical switch with low switching energy for OTDM systems," IEEE Photon. Technol. Lett., vol. 15, pp. 1363-1365, Oct. 2003.

[66] K. Nishimura, R. Inohara, M. Tsurusawa, and M. Usami, "80 Gbit/sec wavelength conversion using MQW electroabsorption modulator in delayed interferometric configuration," Electron. Lett., vol. 39, pp. 792-795, 2003

[67] J. H. den Besten, R. G. Broeke, M. van Geemert, J. M. M. Binsma, F. Heinrichsdorff, T. van Dongen, E. A. J. M. Bente, and M. K. Smit, "An integrated $4 \times 4$-channel multiwavelength laser on InP," IEEE Photon. Technol. Lett., vol. 15, pp. 368-370, Mar. 2003.

[68] H. Ju, S. Zhang, H. de Waardt, G. D. Khoe, and H. J. S. Dorren, Ultrafast All-Optical Switching by Pulse Induced Birefringence in a Semiconductor Optical Amplifier.

[69] T. Akiyama, K. Kawaguchi, M. Sugawara, H. Sudo, M. Ekawa, H. Ebe, A. Kuramata, K. Otsubo, K. Morito, and Y. Arakawa, "A semiconductor optical amplifier with an extremely penalty free output power of $20 \mathrm{dBm}$ achieved with quatumdots," in Proc. Eur. Conf. Optical Communication-Int. Conf. Optical Communication 2003, Rimini, Italy, p. 80

[70] D. G. Deppe and D. L. Huffaker, "Quantum dimensionality, entropy and modulation response of quantum dot lasers," Appl. Phys. Lett., vol. 77, pp. 3325-3327, 2000 .

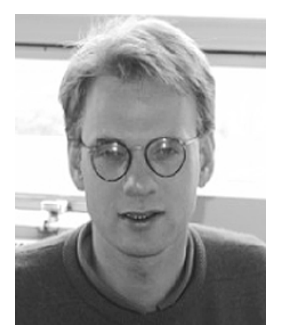

H. J. S. Dorren received the M.Sc. degree in theoretical physics and the Ph.D. degree from Utrecht University, Utrecht, The Netherlands, in 1991 and 1995, respectively.

After a postdoctoral position, he joined Eindhoven University of Technology, Eindhoven, The Netherlands, in 1996, where he currently serves as an Associate Professor. In 2002 he was also a Visiting Researcher at the National Institute of Industrial Science and Technology (AIST), Tsukuba, Japan. His research interests include optical packet switching, digital optical signal processing, and ultrafast photonics.

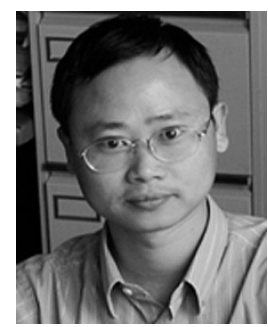

Xuelin Yang was born on Jan. 7, 1967. He received the M.Sc. and Ph.D. degrees from the Department of Applied Physics, Shanghai Jiao Tong University, Shanghai, China. His research fields were mainly in nonlinear optical crystals and devices.

In 1999, he joined Laboratoire Stereochemie de Molecules and Interactions (STIM), Ecole Normale Superieure de Lyon, Lyon, France, as a Postdoctoral Researcher, engaged into research on nonlinear optical materials. In 2001, he became a Postdoctoral Researcher in the Department of Electrical Engineering, Eindhoven University of Technology, Eindhoven, The Netherlands, involved in research into the ultrafast optical signal processing using semiconductor optical amplifiers. He has authored and coauthored more than 40 refereed papers. 


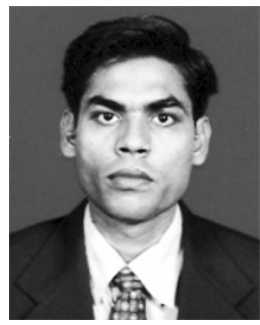

Arvind K. Mishra was born in Varanasi, India, in 1975. He received the M.Sc. degree in physics and the M.Tech. degree in optoelectronics and optical communication from the Indian Institute of Technology, New Delhi, in 1999 and 2001, respectively. $\mathrm{He}$ is currently working toward the Ph.D. degree in the area of ultrafast all-optical signal processing at the Eindhoven University of Technology, Eindhoven, The Netherlands.

During 2001-2002, he was with Philips Research Eindhoven as a research scientist in the ubiquitous communication system group, where he was involved in research on photonic components and systems for in-home networks.

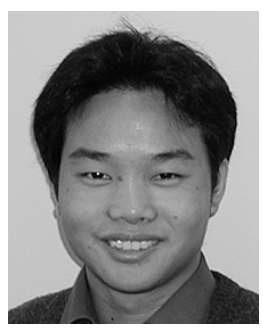

Zhonggui Li was born in Sichuan, China, in 1979. $\mathrm{He}$ received the M.Sc. degree in optical engineering from the University of Electronic Science and Technology of China, Chengdu, China, in 2002. $\mathrm{He}$ is working toward the Ph.D. degree at the Eindhoven University of Technology, Eindhoven, The Netherlands.

His research field is ultrafast optical signal processing.

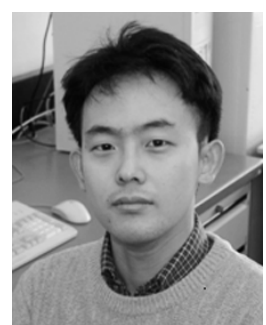

Heongkyu Ju was born in Seoul, Korea, in 1970. He received the B.S. degree in physics and the M.Sc. degree in quantum field theory of elementary particle physics at the Korea University, Seoul, Korea, in 1993 and 1998, respectively, and the Ph.D. degree in condensed matter physics at the University of Oxford, Oxford, U.K. in 2003.

$\mathrm{He}$ is with the Electrooptical Communication Group, Eindhoven University of Technology, Eindhoven, The Netherlands, as a Postdoctoral Researcher. His research interests include ultrafast all-optical switching and memories by using nonlinear ultrafast carrier dynamics in semiconductor optical amplifiers.

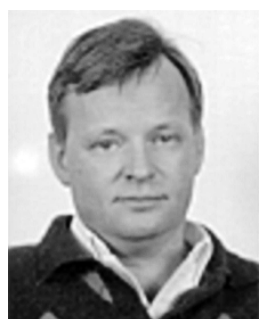

Huug de Waardt was born in Voorburg, The Netherlands, on December 1, 1953. He received the M.Sc.E.E. and Ph.D. degrees from the Delft University of Technology, Delft, The Netherlands, in 1980 and 1995, respectively.

In 1981, he started his professional carreer at PTT Research, Liedschendam, The Netherlands, in the Physics Department, where he worked on the performance issues of semiconductor laser devices. In 1989 he moved to the Transmission Department and became involved in high-bit-rate optical transmission. In 1995, he was appointed as Associate Professor at the Eindhoven University of Technology, Eindhoven, The Netherlands, in the area of high-bit-rate trunk transmission. He coordinated the participation of TUE in ACTS Upgrade, ACTS APEX, and IST FASHION. He has coauthored over 75 scientific contributions. His current research interests are in high-capacity optical transmission, integrated optics, and semiconductor optical amplifiers.

Dr. de Waardt is member of the IEEE Laser and Electro-Optics Society.

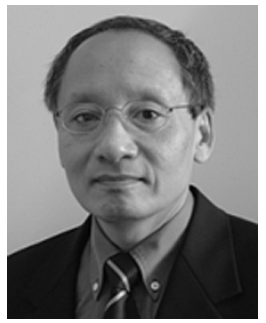

Giok-Djan Khoe (S'71-M'71-SM'85-F'91) was born in Magelang, Indonesia, in 1946. He received the Elektrotechnisch Ingenieur degree (cum laude) from the Eindhoven University of Technology, Eindhoven, The Netherlands, in 1971.

He was a Researcher with the Dutch Foundation for Fundamental Research on Matter (FOM), Laboratory on Plasma Physics, Rijnhuizen, The Netherlands. In 1973, he moved to the Philips Research Laboratories to begin research in the area of optical fiber communication systems. In 1983, he was appointed part-time Professor at Eindhoven University of Technology, becoming a full Professor in 1994. Currently, he is Chairman of the Department of Telecommunication Technology and Electromagnetics (TTE). Most of his work has been devoted to single-mode fiber systems and components. His research programs are centered on ultrafast all-optical signal processing, high-capacity transport systems, and systems in the environment of the users. He has more than 40 U.S. patents and has authored and coauthored more than 100 papers, invited papers, and book chapters. In Europe, he is closely involved in Research Programs of the European Community and in Dutch national research programs, as a participant, evaluator, auditor, and program committee member. $\mathrm{He}$ is one of the founders of the Dutch COBRA University Research Institute. In 2001, he brought four groups together to start a new international alliance called the European Institute on Telecommunication Technologies (eiTT).

Dr. Khoe is an Associate Editor of the IEEE Journal of QuANTUM ELECTRONICS and the appointed President of the IEEE Lasers and Electro-Optics Society (LEOS). Recently, he was General Cochair of the ECOC 2001. He has served the IEEE LEOS organization as a European Representative in the BoG, a Vice President of Finance and Administration, a BoG Elected Member, and a member of the Executive Committee of the IEEE Benelux Section, and was Founder of the LEOS Benelux Chapter. He is the current Junior Past President of LEOS. He received the MOC/GRIN Award in 1997 and was a recipient of the prestigious "Top Research Institute Photonics" grant that is awarded to COBRA in 1998 by the Netherlands Ministry of Education, Culture and Science.

Takasi Simoyama received the B.S. and M.S. degrees in applied physics from the University of Tokyo, Tokyo, Japan in 1993 and 1995, respectively.

He joined Fujitsu Laboratories Ltd. in 1995, where he has been engaged in the research of semiconductor devices for optical communication systems. He joined the Femtosecond Technology Research Association, Tsukuba, Japan, in 2001, where he has been engaged in the research of all-optical switches for optical time domain multiplexing (OTDM) systems.

Hiroshi Ishikawa (SM'94, F'02) was born in Kokura, Japan, in 1946. He received the B.S., M.S., and Dr.Eng. degrees from Tokyo Institute of Technology, Tokyo, Japan, in 1970, 1972, and 1984, respectively.

He joined Fujitsu Laboratories Ltd. in 1972, where he engaged in the research and development of optical semiconductor devices. He invented and developed a Fabry-Pérot laser named the VSB laser, used in TPC-3 undersea cable systems and many trunk lines. He developed distributed feedback (DFB) lasers including modulator-integrated DFB laser for high-bit-rate systems and tunable narrow linewidth DFB lasers for coherent communication systems. He proposed the use of InGaAs substrate for temperature robust $1.3-\mu \mathrm{m}$ lasers, developed the bulk InGaAs growth technology, and realized high $T_{0}$ lasers. He also worked on quantum dot lasers and nonlinear optical devices based on quantum nano-structures. In April 2001, he moved to the Femotosecond Technology Research Association, Tsukuba, Japan. He is now engaged in the research and development of ultrafast optical switches based on optical nonlinearity of quantum nanostructures and organic materials.

Dr. Ishikawa is a Member of the Japan Society of Applied Physics, the Physical Society of Japan, and the Institute of Electronics, Information and Communication Engineers.

Hitoshi Kawashima received the Ph.D. degree in chemistry from Kyoto University, Kyoto, Japan, in 1993.

His research interests include ultrafast optics and its application to device evaluation.

Toshifumi Hasama received the Ph.D. degree in applied physics from Kyoto University, Kyoto, Japan, in 1981.

His research interests include femtosecond solid-state lasers and ultrafast nonlinear optics. 